\title{
High-speed stars: Galactic hitchhikers ${ }^{\star} \star \star$
}

\author{
E. Caffau ${ }^{1}$, L. Monaco ${ }^{2}$, P. Bonifacio ${ }^{1}$, L. Sbordone ${ }^{3}$, M. Haywood ${ }^{1}$, M. Spite ${ }^{1}$, P. Di Matteo ${ }^{1}$, F. Spite ${ }^{1}$, \\ A. Mucciarelli ${ }^{4,5}$, P. François ${ }^{6,7}$, and A. M. Matas Pinto ${ }^{1}$
}

1 GEPI, Observatoire de Paris, Université PSL, CNRS, 5 Place Jules Janssen, 92190 Meudon, France e-mail: Elisabetta.Caffau@obspm.fr

2 Universidad Andres Bello, Departamento de Ciencias Fisicas, Fernandez Concha 700, Las Condes, Santiago, Chile

${ }^{3}$ European Southern Observatory, Casilla 19001 Santiago, Chile

4 Dipartimento di Fisica e Astronomia, Università degli Studi di Bologna, Via Gobetti 93/2, 40129 Bologna, Italy

5 INAF - Osservatorio di Astrofisica e Scienza dello Spazio di Bologna, Via Gobetti 93/3, 40129 Bologna, Italy

${ }^{6}$ GEPI, Observatoire de Paris, Université PSL, CNRS, 77 Av. Denfert-Rochereau, 75014 Paris, France

7 UPJV, Université de Picardie Jules Verne, 33 rue St Leu, 80080 Amiens, France

Received 30 March 2020 / Accepted 24 April 2020

\begin{abstract}
Context. The search for stars born in the very early stages of the Milky Way star formation history is of paramount importance in the study of the early Universe since their chemistry carries irreplaceable information on the conditions in which early star formation and galaxy buildup took place. The search for these objects has generally taken the form of expensive surveys for faint extremely metal-poor stars, the most obvious but not the only candidates to a very early formation.

Aims. Thanks to Gaia DR2 radial velocities and proper motions, we identified 72 bright cool stars displaying heliocentric transverse velocities in excess of $500 \mathrm{~km} \mathrm{~s}^{-1}$. These objects are most likely members of extreme outer-halo populations, either formed in the early Milky Way build-up or accreted from since-destroyed self-gravitating stellar systems.

Methods. We analysed low-resolution FORS spectra of the 72 stars in the sample and derived the abundances of a few elements. Despite the large uncertainties on the radial velocity determination, we derived reliable orbital parameters for these objects.

Results. The stars analysed are mainly slightly metal poor, with a few very metal-poor stars. Their chemical composition is much more homogeneous than expected. All the stars have very eccentric halo orbits, some extending well beyond the expected dimension of the Milky Way.

Conclusions. These stars can be the result of a disrupted small galaxy or they could have been globular cluster members. Age estimates suggest that some of them are evolved blue stragglers, now on the subgiant or asymptotic giant branches.
\end{abstract}

Key words. stars: abundances - Galaxy: abundances - Galaxy: evolution - Galaxy: formation - Galaxy: kinematics and dynamics Galaxy: halo

\section{Introduction}

The results of the Gaia satellite (Gaia Collaboration 2016) and especially those of its second data release (DR2; Gaia Collaboration 2018; Arenou et al. 2018) have revolutionised our understanding of the build-up of the Galaxy. In particular it now appears that the halo stars ${ }^{1}$ in the Gaia catalogue are dominated by stars on disc orbits and stars with low rotational angular momentum that are interpreted as the result of a major merger with a massive satellite (Belokurov et al. 2018; Haywood et al. 2018; Helmi et al. 2018; Di Matteo et al. 2019). We refer to this satellite as Gaia-Sausage-Enceladus as done by Di Matteo et al. (2020), and its mass may have been in excess of $10^{10}$ solar masses (Belokurov et al. 2018; Mackereth \& Bovy 2020). The stars on disc orbits may be prograde or retrograde, and their excursion about the Galactic plane may be as

\footnotetext{
* Chemical and kinematic data are only available at the CDS via anonymous ftp to cdsarc.u-strasbg.fr (130.79.128.5) or via http://cdsarc.u-strasbg.fr/viz-bin/cat/J/A+A/638/A122

$\star \star$ Based on observations made with ESO Telescopes at the La Silla Paranal Observatory under programme ID 104.D-0259.

1 We loosely define halo stars as those with a total speed in excess of $180 \mathrm{~km} \mathrm{~s}^{-1}$ (see e.g. Di Matteo et al. 2019).
}

large as a few kpc (thick disc) or as small as a few hundred pc (thin disc). Quite unexpectedly, the most metal-poor star known, SDSS J1029+1729, is on a thick-disc orbit (Caffau 2018; Sestito et al. 2019; Di Matteo et al. 2020). Another extremely metal-poor star 2MASS J18082002-5104378 (Meléndez et al. 2016) with an iron abundance $[\mathrm{Fe} / \mathrm{H}]=-3.84$ (Spite et al. 2019) is on a thin-disc orbit (Schlaufman et al. 2018; Sestito et al. 2019; Di Matteo et al. 2020). More generally it now appears that when a sample of stars is chemically selected as metal poor there is always a significant fraction that is on disc orbits (see e.g. Venn et al. 2020). At the same time there is no evidence for the presence of a classical halo formed in situ as envisaged in the seminal Eggen et al. (1962) paper (Haywood et al. 2018; Di Matteo et al. 2019). The scenario in which the halo is formed from a combination of in situ early star formation and the accretion of stars and gas from tidally destroyed substructures, the latter dominating at large galactocentric radii (Tissera et al. 2013), needs to be deeply revised (see also Gallart et al. 2019). The number of significant fusions experienced by the Milky Way in the few Gyr of its existence is still debated (Myeong et al. 2019; Kruijssen et al. 2019; Reina-Campos et al. 2020).

In the Gaia data there is still space to identify stars that are the result of minor mergers, and a possible strategy for 
identifying these stars is to select stars with very high kinetic energy. It has been known for a long time that stars with high radial velocities or transverse velocities, which were once considered to belong to the Galactic halo, tend to have low metallicities (Roman 1950). Thus, in the past many observers targeted stars in high-velocity catalogues (Roman 1955; Giclas et al. 1971; Lee 1984) in order to find metal-poor halo stars. One of the first two metal-poor stars detected (HD 19445; Chamberlain \& Aller 1951) was in fact in the Roman (1955) catalogue of high-velocity stars.

In recent years a number of surveys explicitly targeted at the discovery of low-metallicity stars have been employed to search in large spectroscopic or photometric surveys for these very rare primordial stars (usually, albeit misleadingly perhaps, identified with extremely iron-poor stars). Among other, our group developed (Ludwig et al. 2008) and successfully employed a selection tool able to detect candidate extremely metal-poor and extremely iron-poor dwarf stars in the low-resolution Sloan Digital Sky Survey (SDSS; York et al. 2000) spectra for subsequent follow-up (see e.g. Caffau et al. 2013). Among other results, this allowed the discovery of one of the only two non-CN enhanced ultra-metal-poor stars, SDSS J102915+172927 $([\mathrm{Fe} / \mathrm{H}]=-4.99$; Caffau et al. 2011a), which still holds the record for the object with the lowest total metallicity (given that most extremely $\mathrm{Fe}$ poor stars also show extreme $\mathrm{C}, \mathrm{N}, \mathrm{O}$ enhancements).

The quality and richness of the Gaia DR2 catalogue allows us to revive the classical strategy of kinematical selection, although our objectives are more ambitious than those of observers in the past. We do not simply want to find metalpoor stars, but we want to understand how the population(s) with extreme kinetic energy fit in the formation of the Milky Way. For this reason we want to assemble a chemical and kinematical census of these extreme objects that is as complete as possible. In contrast to most deep, targeted surveys, Gaia covers the full sky. The spectrographic capabilities in DR2 are not able to identify very or extremely metal-poor objects, but thanks to the accurate distances and proper motions of nearby stars delivered by Gaia it allows the transverse velocity of a large sample of relatively bright targets to be derived.

In the GaiaDR2 catalogue, we selected stars with large transverse velocity, expecting them to be old stars on extreme, highly elliptic bound orbits with apogalacticon well into the sparsely studied outer halo. Such objects might either form in situ or be accreted from tidally dissolved substructures (dwarf galaxies, stellar clusters). Outer-halo accreted stars, in particular, have a high likelihood of coming from low-mass or ultra-faint dwarf galaxies (Tissera et al. 2013), so should generally be metal poor, and have a relatively high fraction of extremely metal-poor objects, crucial to understanding the early phases of the chemical evolution of the Universe. While this method is less likely to discover ultra-metal-poor objects with respect to targeted surveys, it has a few major advantages:

- It selects very bright objects, whose observations is much less time-consuming, while yielding spectra of much higher quality. This allows us to design observations for these objects as a bad weather or bright time filler programme.

- Instead of selecting a priori on low metallicity (that can be, but is not necessarily, a marker of a very early origin), it targets stars that are likely kinematically associated with the ancient, poorly studied outer-halo population, thus biasing the sample in a very different way.

- It efficiently exploits the less favourable observing conditions at the Very Large Telescope (VLT, i.e. thick clouds, bright time, bad seeing) to mine the incredibly large reservoir of

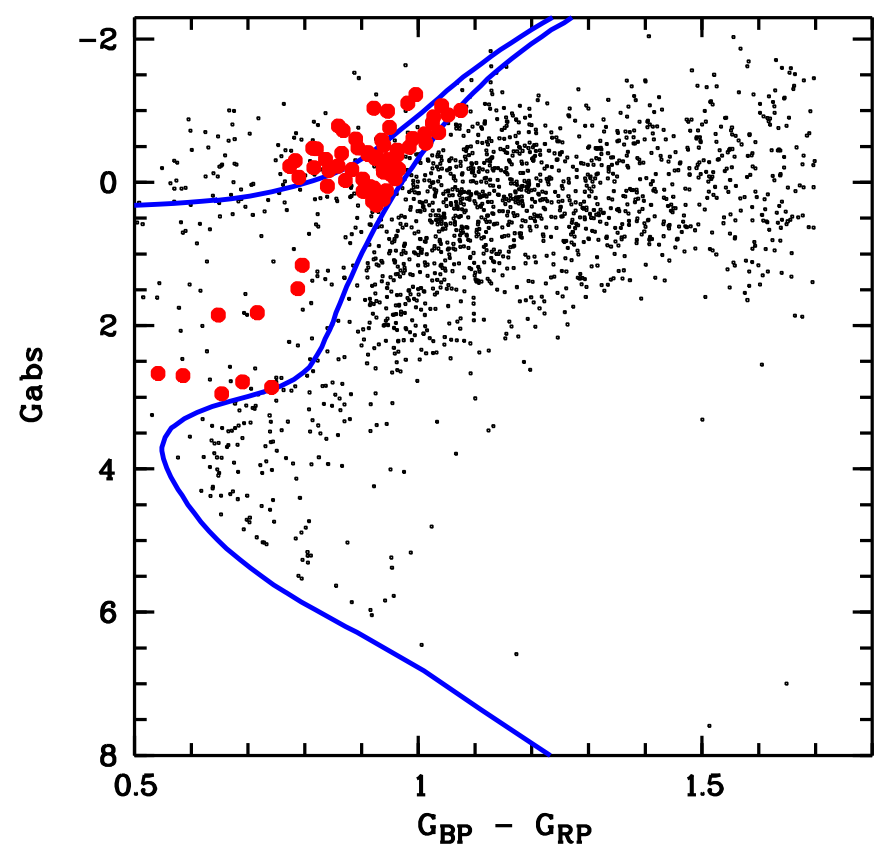

Fig. 1. Gaia colour-magnitude diagram of all stars selected for observation in ESO period 104. The filled red dots are the 72 stars observed. The blue solid line is a PARSEC isochrone (Bressan et al. 2012; Marigo et al. 2017) of metallicity -2.0 and age of $12 \mathrm{Gyr}$, to guide the eye.

unstudied bright cool stars; the whole sample, except for three stars, consists of stars with no reference whatsoever associated with them in SIMBAD (Wenger et al. 2000).

\section{Selection}

From the GaiaDR2 catalogue, we selected stars with transverse velocity higher than $500 \mathrm{~km} \mathrm{~s}^{-1}$, in the $\mathrm{G}$ magnitude range 14-14.5. Further constraints were put on right ascension to ensure observability in European Southern Observatory (ESO) period $104(0 \mathrm{~h} \leq \alpha \leq 16 \mathrm{~h}$ or $\alpha \geq 20)$ and on declination $(\delta \leq-25)$ to privilege a south pointing. The latter constraint was set in order to ensure that the VLT could observe our targets even in the event of fairly strong northern wind. In this way we were able to ensure observations of stars that were not too far away, and as a consequence with relatively small uncertainties on parallaxes and proper motions, and of bright objects for an $8 \mathrm{~m}$ class telescope, allowing good quality observations even in bad weather conditions. This selection provided us with 1824 possible targets. Finally we selected for observation a set of "blue" stars in the hope that we would be selecting the most metal-poor population. The Gaia colour-magnitude of all the possible targets is shown in Fig. 1. In the figure the observed stars are highlighted and a metalpoor old isochrone is shown for reference. All 72 stars shown in Fig. 1 were observed during ESO period 104

From Fig. 1 it is clear that with our selection we favoured (i) asymptotic giant branch (AGB) stars over red giant branch (RGB) stars, (ii) metal-poor stars over solar metallicity stars, and (iii) hotter stars belonging to younger populations. There is also another bias in favour of young stars. Because of our limit on the apparent magnitude of the observed stars, the more luminous the observed stars are, the larger the explored volume is (the greater the distance from the Sun, the higher the luminosity of a star of given apparent magnitude). This is known as the Malmquist bias (Malmquist 1925): the absolute magnitude of a population 
is biased towards higher brightness objects, when the population is drawn from a sample, by applying an apparent magnitude limited selection.

In the kinematical and chemical investigations, we assumed that all stars are single. Spectroscopically and photometrically all stars in the sample, except perhaps one (GHS36; see Sect. 4.3), are compatible with the contribution of a single component. However, a binarity of some of the stars analysed here could affect the astrometric parameters provided by Gaia as an overestimation on the uncertainties (see e.g. Kervella et al. 2019; Penoyre et al. 2020) and as a consequence could affect the kinematic results.

The stars we observed are not known in the SIMBAD astronomical database, except for three objects, which are discussed in the appendix.

- GHS29: Gaia DR2 5188812082642658944, also known as CSTAR 9171, a variable star of RR Lyr type.

- GHS46: Gaia DR2 4629181692264635520, also known as $V^{*} \mathrm{Z}$ Men, a variable star of RR Lyr type.

- GHS63: Gaia DR2 2321153334969276160, also known as CTLGM 5192.

\section{Observations}

The stars were observed with FORS2 (Appenzeller et al. 1998) at VLT-ESO, during the programme ID 104.D-0259, $1 \mathrm{~h}$ OB per star, translated in $2762 \mathrm{~s}$ of exposure time. The observations were done with GRISM $600 \mathrm{~B}+22,0$ ' 28 wide slit providing a resolving power of about 2800 in the spectral range $330-621 \mathrm{~nm}$, with $1 \times 1$ binning of the CCD $\left(0.075 \mathrm{~nm} \mathrm{pixel}^{-1}\right)$. Of the $72 \mathrm{OBs}$, 66 are classified as A and 6 as B. The programme was executed in service mode, as a filler, so all the observations were done in extreme condition of Moon illumination and/or air mass. Of the sample of 72 stars, 23 stars were observed in dark time, 10 in grey time, and 39 in bright time. The distance from the Moon was sometimes small (up to $33^{\circ}$ ). In the data reduction, done with the ESO pipeline, the sky was subtracted with the option "optimal subtraction" to remove the sky contribution from the spectrum. The stars are bright enough to allow a good signal-tonoise ratio $(\mathrm{S} / \mathrm{N}$ at $520 \mathrm{~nm}$ of the order of 90 for all stars, with the lower value of about 70 for GHS59 and few of the subgiant stars with about 100). The spectra of two of the most metal-poor stars in the sample are shown in Fig. 2.

\section{Analysis}

\subsection{Radial velocity and kinematics}

For each star we computed an ATLAS 9 (Kurucz 2005) model with the stellar parameters we derived. We computed a synthetic spectrum with SYNTHE (see Kurucz 2005; Sbordone et al. 2004) and the radial velocities were derived by templatematching (see e.g. Caffau et al. 2020). Using the star's coordinates, radial velocities, Gaia Dr2 distances, and proper motions we characterised the stellar orbital parameters. For this purpose, we used the GalPot $\operatorname{code}^{2}$ (Dehnen \& Binney 1998) together with the best fit model from McMillan (2017). We derived thus the star's coordinates and velocity components in the galactocentric cylindrical $\left(R, z, \phi, v_{R}, v_{Z}, v_{\phi}\right)$ and Cartesian systems $\left(X, Y, Z, v_{X}, v_{Y}, v_{Z}\right)$. We further characterised the orbit deriving the minimum and maximum cylindrical $\left(R_{\min }, R_{\max }\right)$ and spherical $\left(r_{\min }, r_{\max }\right)$ radii, the maximum height above the galactic plane $\left(Z_{\max }\right)$, the total energy $(\mathrm{E})$, the $z$-component of the

\footnotetext{
2 https://github.com/PaulMcMillan-Astro/GalPot
}

angular momentum $\left(L_{Z}\right)$, and the eccentricity (calculated as $\left.e=\left(r_{\max }-r_{\min }\right) /\left(r_{\max }+r_{\min }\right)\right)$. We used the default settings of GalPot, where the solar peculiar motion is the value measured by Schönrich et al. $\left(2010, U_{\odot}=11.1, V_{\odot}=12.24, W_{\odot}=7.25 \mathrm{~km} \mathrm{~s}^{-1}\right)$ and $R_{0}=8.21 \mathrm{kpc}, Z_{0}=0.014 \mathrm{kpc}$, and $v_{\phi}\left(R_{0}\right)=233.1 \mathrm{~km} \mathrm{~s}^{-1}$ for the Sun. It should be noted that in the GalPot conventions the Sun rotates in the negative $\phi$ direction, and thus prograde motion corresponds to negative values of $v_{\phi}$ and $L_{Z}$. The major contribution on the speed of these stars is due to the transversal velocity; the radial velocity plays a minor role in the total speed of the stars. In this way, also with the large uncertainties on the radial velocity determinations due to the flections of FORS2 combined with the lack of telluric lines in the spectral range, we are confident with regard to the nature of the stellar kinematic. To provide more solid results, we computed the orbital parameters again by using $V_{\text {rad }} \pm \sigma$ and the general behaviour does not change.

Five stars in the sample (GHS22, GHS33, GHS37, GHS58, and GHS64) return positive total energies E, and are thus unbound in the McMillan (2017) potential (total virial mass $1.3 \times 10^{12} M_{\odot}$ ), while all the other stars belong to the Galactic halo, with the majority on retrograde orbits (44/72) and only $32 \%(23 / 72)$ on prograde orbits. Integration backwards of the orbits over $13 \mathrm{Gyr}$ using the $\mathrm{GalPy}^{3}$ code (Bovy 2015) with the MWPotential2014 potential multiplied by 1.63 in order to match the same mass of the McMillan (2017) best fit model, supports the notion that these stars are in fact unbound to the Galaxy. Masses between $1.5 \times 10^{12} M_{\odot}$ (for star GHS33) and $2.0 \times 10^{12} M_{\odot}$ (for star GHS58) would be required to bound the stars although with apocentres in excess of $500 \mathrm{kpc}$. Of these five stars, only GHS64 has a direction of motion which is broadly compatible with coming from the Andromeda region. On the other hand, none is compatible with an origin in the Magellanic Clouds.

In Figs. 3 and 4 the orbital characteristics are presented. In the two figures the stars shown are (i) prograde stars as red triangles, (ii) retrograde stars as open black symbols, and (iii) the five unbound stars, when present, as filled black squares. The majority of the stars have very eccentric orbits, with 57 stars having an eccentricity higher than 0.9. Of the five unbound stars, three are prograde and two retrograde. The maximum (in plane) distance from the Galactic centre, $R \max$ is very large for some stars, larger than the Galactic radius. The Toomre diagram (upper right panel in Fig. 4) is comparable to the same diagram in Di Matteo et al. (2020, see their Fig. 3, upper left panel). The fraction of prograde and retrograde stars was different in Di Matteo et al. (2020), being 14/42 retrograde and 28/42 prograde. The stars in Di Matteo et al. (2020), selected only for their metallicity, were observed during the ESO large programme 165.N-0276 (Cayrel et al. 2004) and analysed in several papers (see e.g. Spite et al. 2005; François et al. 2007; Bonifacio et al. 2009).

\subsection{Discussion on the parallax zero point}

The GaiaDR2 astrometric solution is described at length in Lindegren et al. (2018) and the issue of a zero-point in the Gaia parallaxes is discussed in detail. An instrument like Gaia is designed to give absolute parallaxes; however, it is still prone to systematic errors, in particular to the precise value of the basic angle, which is the angle between the two primary mirrors. For Gaia this angle has been discovered to vary in time, thanks to an on-board metrology interferometer (the Basic Angle Monitor,

\footnotetext{
https://github.com/jobovy/galpy
} 


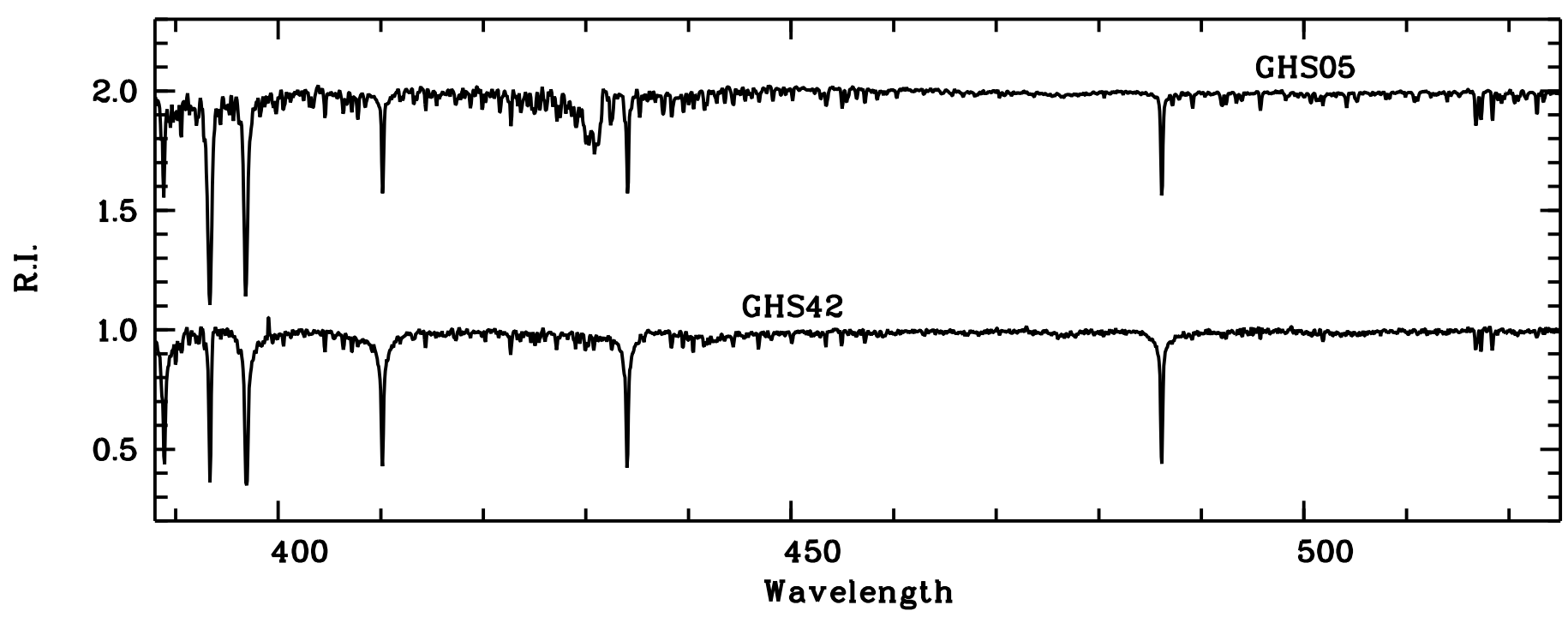

Fig. 2. Spectra of the two most metal-poor stars. Both spectra have been normalised, and the spectrum of GHS05 has been shifted up by 1 , for easy visualisation.
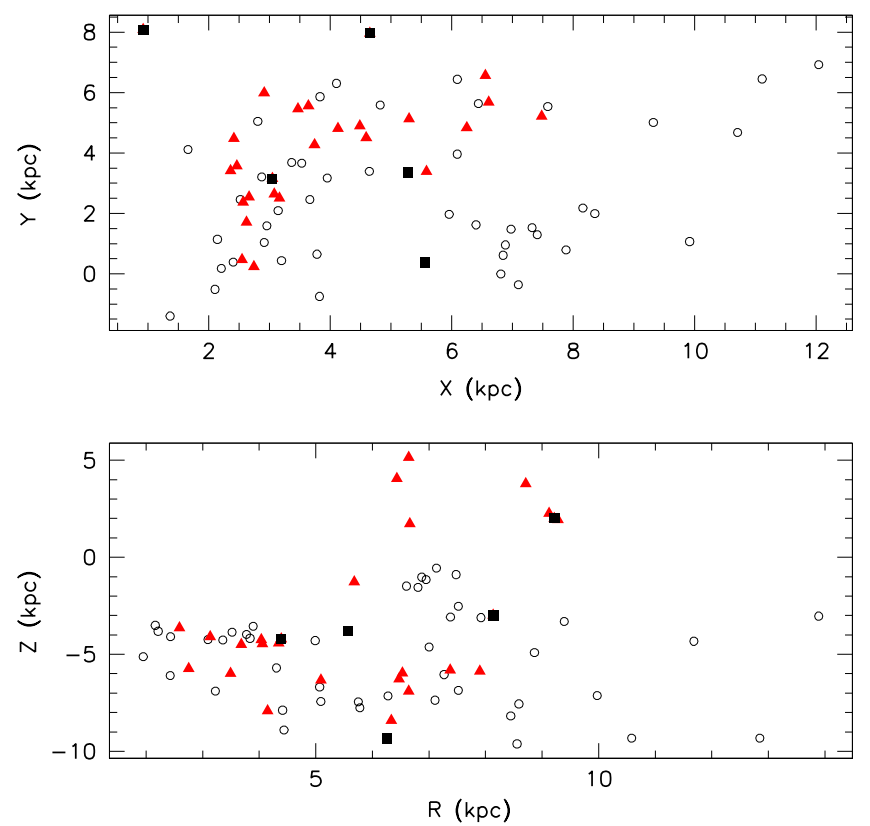

Fig. 3. Portion of the stars in the $\mathrm{XY}$ and RZ planes. The stars are classified as prograde stars (red triangles), retrograde stars (open black symbols), and unbound stars (filled black squares).

BAM). The variations of basic angle as measured by the BAM are taken into account in the Gaia global calibration. From the parallax distribution of 556869 sources classified as active galactic nuclei, therefore at distances where their true parallax should be zero within the sensitivity of Gaia, Lindegren et al. (2018) derive a global zero point of -0.029 mas. While within the accuracy of DR2 this is a statistically robust zero point, Lindegren et al. (2018) warn that "the actual offset applicable for a given combination of magnitude, colour, and position may be different by several tens of $\mu$ as". In our case the magnitudes of our objects are several magnitudes brighter than the bulk of the sources used to determine the global zero point and the colours of our stars are redder. To look for a more relevant comparison sample, we consider the results of Arenou et al.
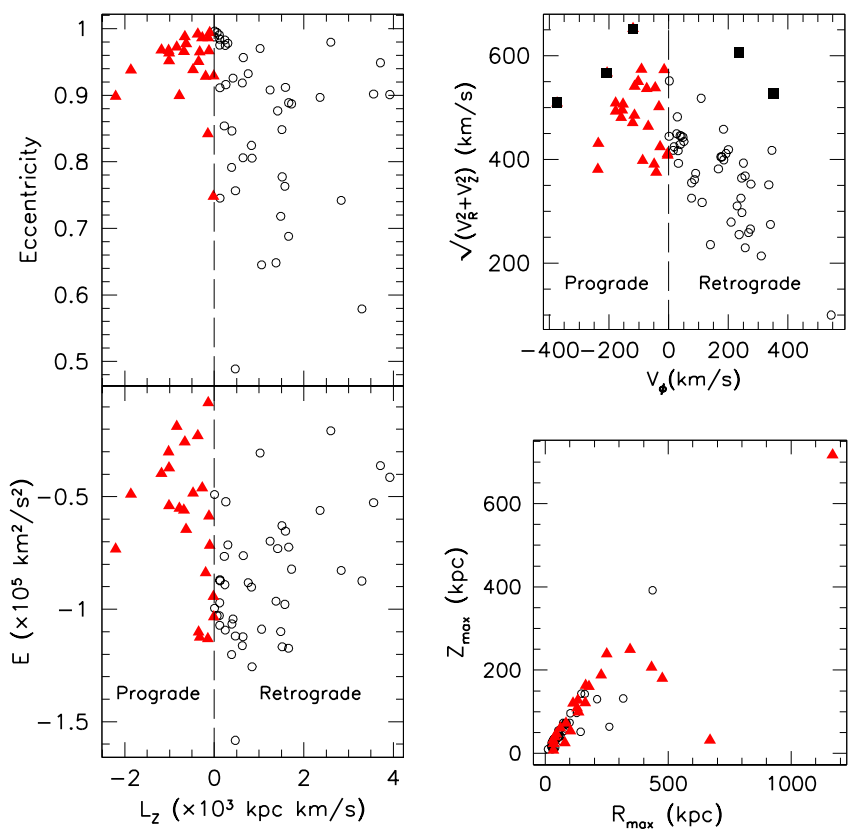

Fig. 4. Orbital parameters for the stars. Symbols are as in Fig. 3.

(2018), who, compared the Gaia parallaxes for star members of 200 open clusters of known distance and derived the offsets, $\Delta \varpi=-0.067 \pm 0.12$ mas and $\Delta \varpi=-0.064 \pm 0.17$ mas, for two different catalogues of open clusters. It should be kept in mind that the error associated with these two estimates of the zero point is not statistical in nature, but systematic, deriving from two very different systematics that affect the Gaia parallaxes and the determiniation of the open cluster distances. Therefore, the error on the zero point cannot be divided by the square root of the number of clusters.

Considering the uncertainty on the Gaia parallax zero point and that the stars lie in different directions in the sky, we considered that it is a more conservative choice not to apply any zero point. Nevertheless, we investigated the effect of applying a zero point correction to our sample of stars. For this test we choose -0.065 mas, which is an average of the two values found 
by Arenou et al. (2018) for open clusters. When this zero-point is applied to the Gaia DR2 data, all the distances of our stars become smaller with respect to the value shown in the figures. The effect is first noticeable in the colour-magnitude diagram. The luminosity of all the giant stars becomes smaller by up to two magnitudes. For the majority of the stars their position in the colour-magnitude diagram is no longer consistent with any isochrone of a metallicity consistent with the spectroscopic metallicity.

The second noticeable effect is on the kinematics. Because the stars are placed at smaller distances their transverse velocities become smaller thus all 72 stars would now be bound. The conclusions derived would be approximately the same even with this assumption. The stars remain on high-eccentricity halo orbits with a significant fraction of retrograde stars.

\subsection{Stellar parameters}

To derive the stellar parameters, we used the Gaia DR2 catalogue. With the parallax provided by Gaia DR2, we first derived the absolute $\mathrm{G}$ magnitude, and then by comparing the BP-RP colour and the $\mathrm{G}$ absolute magnitude to PARSEC ${ }^{4}$ isochrones (Bressan et al. 2012; Marigo et al. 2017) of a certain metallicity, we derived the stellar parameters. The Gaia photometry was dereddened by using the maps by Schlegel et al. (1998). With the derived stellar parameters, we ran our pipeline MyGIsFOS (Sbordone et al. 2014) to derive the chemical composition of each star. When the derived Fe abundance was not within about 0.25 dex of the metallicity of the isochrone used to derive the stellar parameters, new parameters were obtained by using isochrones of closer metallicity, and provided as input to MyGIsFOS.

Two of the three stars already investigated in the literature, GHS29 and GHS46, are classified as RR Lyr in the Gaia DR 2 catalogue. We compared $\mathrm{H} \beta$, which is available in the wavelength range observed, to synthetic spectra computed with the stellar parameters derived from the colours. The agreement is good. We made some further checks on these two stars:

- GHS29 The effective temperature $\left(T_{\text {eff }}=6100 \mathrm{~K}\right)$ we derived is more than $100 \mathrm{~K}$ hotter than $5885.0 \mathrm{~K}$ provided in the Gaia catalogue. The metallicity of $-1.22 \pm 0.22$ provided in the Gaia DR2 RR Lyr catalogue is in good agreement with the value $[\mathrm{Fe} / \mathrm{H}]=-1.38 \pm 0.30$ we derived. To verify the reliability of our analysis, we derived the stellar parameters by using the Gaia epoch photometry in correspondence with the phase during the FORS observation, and derived $T_{\text {eff }}=6113 \mathrm{~K}$ and $\log g=2.74$, very close to the value derived from the average photometry provided by Gaia DR2 $(6100 \mathrm{~K} / 2.75)$ and used in this analysis.

- GHS46 The effective temperature we derived ( $T_{\text {eff }}$ $=5976 \mathrm{~K})$ is in perfect agreement with $5950.997 \mathrm{~K}$ provided in the Gaia catalogue. The metallicities we derived for this star $([\mathrm{Fe} / \mathrm{H}]=-2.54 \pm 0.27)$ is in good agreement with the metallicity of $-2.22 \pm 0.22$ provided by the Gaia DR2 RR Lyr catalogue. When using the Gaia epoch photometry corresponding to the phase of the observation to derive the stellar parameters, we find $T_{\text {eff }}=5931 \mathrm{~K}$ and $\log g=2.47$, very close to the value adopted here and derived from the Gaia DR2 photometry (5976 K/2.50).

On the other hand, we suspected that the star GHS36 is also a RR Lyr: the observed Gaia photometry put it far from the isochrone of the metallicity derived from the analysis. This star from the photometry should be young and more metal poor. When comparing the $\mathrm{H} \beta$ wings to a synthetic spectrum, the star appears hotter than derived. We do not provide detailed abundances for this star, but only a probable metallicity.

\footnotetext{
4 http://stev.oapd.inaf.it/cgi-bin/cmd
}

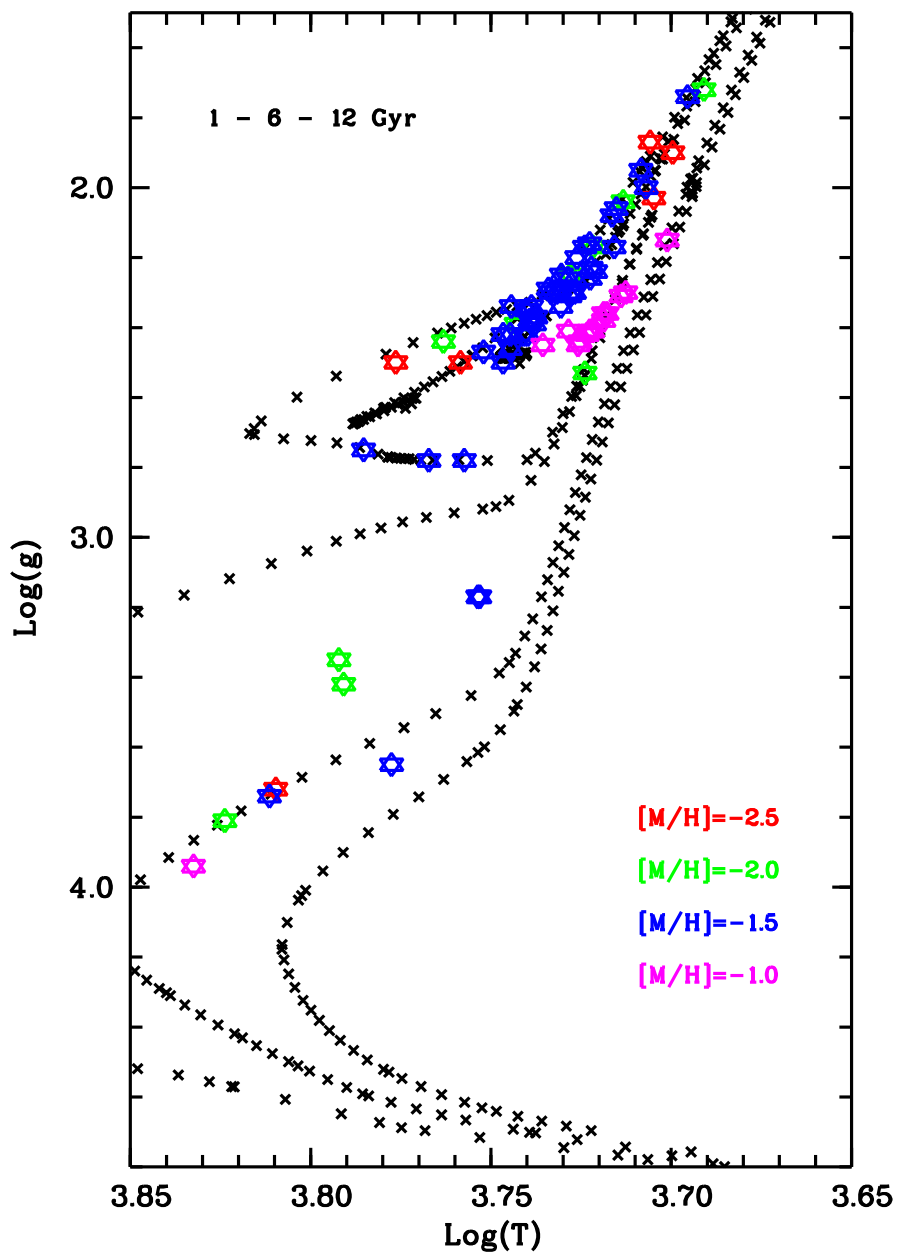

Fig. 5. Hertzsprung-Russell diagram comparing the observed stars with isochrones of metallicity $[\mathrm{M} / \mathrm{H}]=-1.5$, at three ages $(1,6$, and $12 \mathrm{Gyr})$, the value closest to the metallicity derived for the largest fraction of the stars.

In Fig. 5 the parameters we derived for all the stars in the sample are compared to isochrones of $[\mathrm{M} / \mathrm{H}]=-1.5$ for three ages, and in Fig. 6 the Gaia DR2 photometry of our sample of stars is compared to the isochrones. In Table 1 the stellar parameters are presented; as solar Fe abundance we applied $\mathrm{A}(\mathrm{Fe})=7.52$ by Caffau et al. (2011b, see also Table 2 ).

From Fig. 6 we can deduce that the stars appear younger that expected from their relatively low metallicity. This effect could be the result of an overcorrection of the reddening. We then compared the photometry not corrected for reddening to the isochrones. Of course the stars move towards older ages, but the problem of stars that are too young for the metallicity still persists. The two known RR Lyr in the sample, GHS29 and GHS46, have to be old stars to be in this phase of their evolution, but to derive their stellar parameters we had to accept a young age. RR Lyr are variable stars, so these stars cannot be used to discuss the reliability of the ages. On the other hand, the other stars in the sample can hardly be RR Lyr type because their parameters (except for few stars) are not compatible with this stage of stellar evolution.

\subsection{Abundances}

With the code MyGIsFOS (Sbordone et al. 2014), we derived the chemical composition of the stars of our sample. 


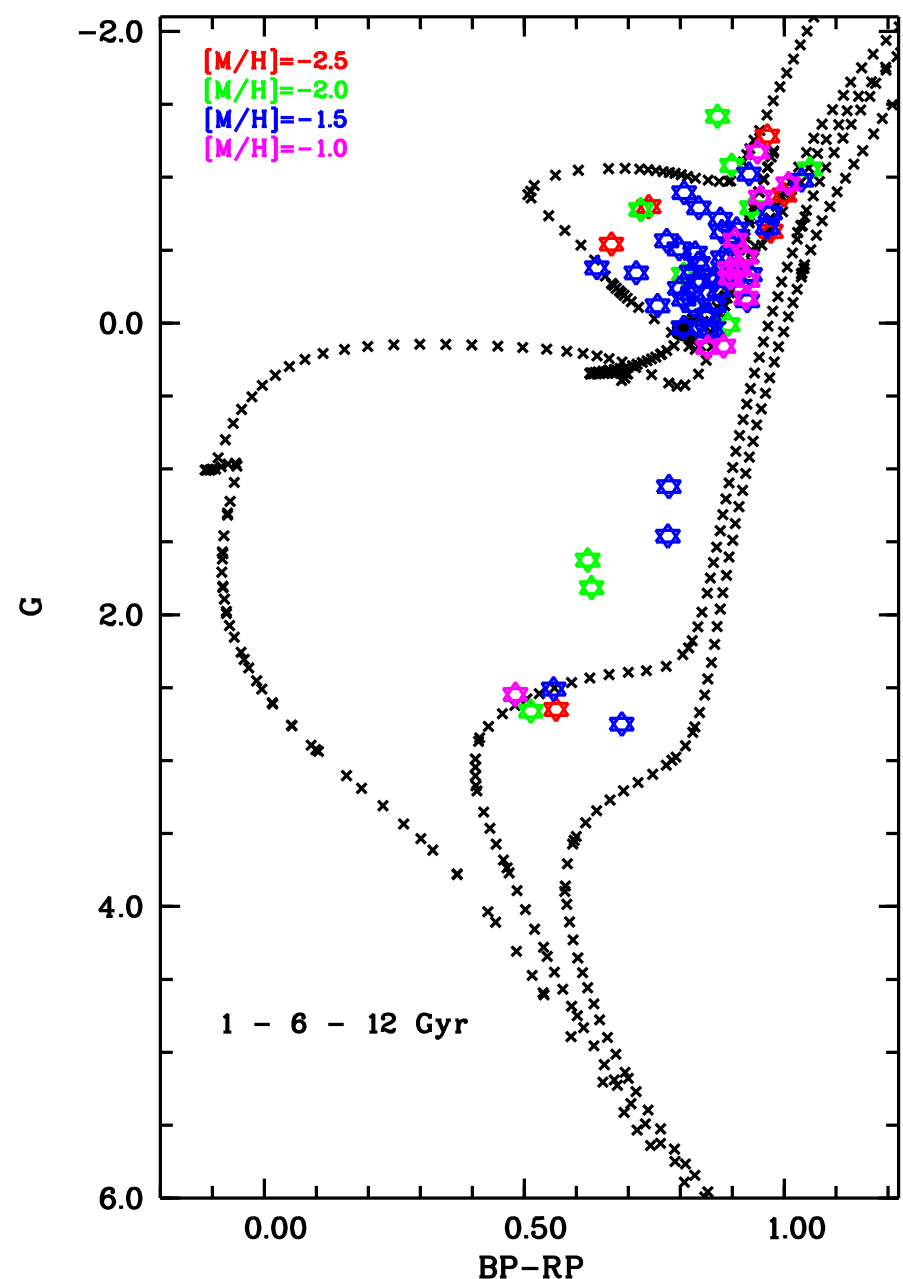

Fig. 6. Hertzsprung-Russell diagram of G Gaia vs. BP-RP, comparing the observed stars with isochrones of metallicity $[\mathrm{M} / \mathrm{H}]=-1.5$, for three ages $(1,6$, and $12 \mathrm{Gyr})$. The colour-coding for the stars defines the metallicity of the isochrone used to derive the stellar parameters. The blue symbols correspond to the stars that used isochrones of $[\mathrm{M} / \mathrm{H}]=-1.5$, as in the plot.

MyGIsFOS compares the selected features to a pre-computed grid of synthetic spectra, which we computed with the spectralsynthesis code SYNTHE (see Kurucz 2005; Sbordone et al. 2004) based on 1D model atmospheres we computed with ATLAS 12 (Kurucz 2005), and which makes a $\chi^{2}$ minimisation. We could derive the abundances of $\mathrm{Fe}$ (by using Fe I features, and in few cases also from Fe II features), $\mathrm{Mg}, \mathrm{Ca}$, $\mathrm{Ti}$ (both from $\mathrm{Ti}$ I and Ti II features), and Ba for all or most of the stars. We also derived $\mathrm{Ni}$ and $\mathrm{Sr}$ abundances for a subsample of stars (6 and 22, respectively). The $\mathrm{Fe}$ abundance we derive for our sample is in the range $-2.61 \leq[\mathrm{Fe} / \mathrm{H}] \leq-0.95$.

In Fig. 7 the comparison of the abundances derived by MyGIsFOS from neutral and single ionised lines of $\mathrm{Ti}$ and $\mathrm{Fe}$ is shown. The agreement is generally very good. The higher $\mathrm{A}(\mathrm{Ti})$ value derived from Ti II lines with respect to that from Ti I lines is explained by NLTE effects (see Mashonkina et al. 2016).

In Fig. 8, the abundances of the two analysed $\alpha$-elements, $\mathrm{Mg}$ and $\mathrm{Ca}$, are shown. The reference solar abundances for this figure and the others are listed in Table 2 . The sample shows a very homogeneous $\alpha$-enhancement, even though that the analysis is based on low-resolution spectra. As the average $[\mathrm{Mg} / \mathrm{Fe}]$ for the complete sample (71 stars) we derived $\langle[\mathrm{Mg} / \mathrm{Fe}]\rangle=+0.42 \pm 0.11$ and for the 70 stars for which we have $\mathrm{A}(\mathrm{Ca})$ determination from $\mathrm{Ca}$ I lines $\langle[\mathrm{Ca} / \mathrm{Fe}]\rangle=+0.31 \pm 0.10$. To make this statement stronger, we compared our sample to the FORS-Pristine sample (Caffau et al. 2020), which is a sample of similar metallicity stars, observed with the same spectrograph. These stars (open blue squares in the figure) generally show a larger scatter in $[\mathrm{Mg} / \mathrm{Fe}]$ and $[\mathrm{Ca} / \mathrm{Fe}]$, overall showing several stars with no evidence for the typical $\alpha$-enhancement expected for the Galactic metal-poor stars. This situation is not seen in the sample of stars presented here.

For six stars we were able to derive the abundance of Ni from one single feature. In Fig. 9 these results are compared to literature values, and we see that this sample is completely compatible with stars of similar metallicity.

In Fig. 10 the abundances of the two heavy elements are shown. For the stars that also have a detection in $\mathrm{Sr}$ and $\mathrm{Ba}$, they behave as normal Galactic metal-poor stars. In Fig. 11, $[\mathrm{Sr} / \mathrm{Fe}]$ and $[\mathrm{Ba} / \mathrm{Fe}]$ are plotted versus $[\mathrm{Fe} / \mathrm{H}]$, and are compared to the sample by Caffau et al. (2020) and to literature values collected by Frebel (2010). In Fig. 11, the most metal-poor stars in our sample $([\mathrm{Fe} / \mathrm{H}]<-2)$ are compared to the stars with $[\mathrm{Fe} / \mathrm{H}]<-2$ in Caffau et al. (2020) and to literature values for extremely metal-poor stars.

One star, GHS48, shows a high $[\mathrm{Ba} / \mathrm{Fe}]$ value and no $\mathrm{Sr}$ determination due to the poor reproduction of the fit on the SrII line, and the abundance determination was rejected by MyGIsFOS because the probability was below threshold (for details, see Sbordone et al. 2014). The other star rich in $\mathrm{Ba}(\mathrm{GHS} 65,[\mathrm{Ba} / \mathrm{Fe}]=1.03)$ is a carbon enhanced metal-poor $(\mathrm{CEMP}) \operatorname{star}([\mathrm{C} / \mathrm{Fe}]=1.21)$, but its $\mathrm{Sr}$ is just slightly enhanced $([\mathrm{Sr} / \mathrm{Fe}]=0.43)$.

The $G$-band is generally strong for cool stars, so for the majority of the stars (55 stars) we were able to derive the $\mathrm{C}$ abundance. For the others, we provide an upper limit. Two stars (GHS65 and GHS69) are CEMP ([C/Fe] > 1). GHS65 is a CEMP star also rich in $\mathrm{Ba}([\mathrm{Ba} / \mathrm{Fe}]=1.03)$. It is worth repeating that several evolved stars in this sample are probably AGBs, so that part of their $\mathrm{C}$ has been converted into $\mathrm{N}$.

To derive the $\mathrm{C}$ abundances or upper limits, by using SYNTHE and the model atmosphere with the parameters of the star, we computed a grid of synthetic spectra varying in $\mathrm{A}(\mathrm{C})$. With a $\chi^{2}$ minimisation, we derive $\mathrm{A}(\mathrm{C})$. When $\mathrm{A}(\mathrm{C})$ is known, from the $\mathrm{CN}$ band at $380 \mathrm{~nm}$, it is possible to derive the $\mathrm{N}$ abundance with the same technique. This $\mathrm{CN}$ band is much weaker than the $G$-band, and at this resolution is hardly detectable; however, for a subsample of stars (35 stars, generally the cooler and the most metal-rich in the sample) we were able to derive $A(N)$. We have so many $\mathrm{N}$ detections because these stars are mostly cold, but also because some of our stars are AGBs and their N content is enhanced. In Fig. 12, the $\mathrm{C}$ abundance derived from the $G$-band is plotted as a function of the stellar $[\mathrm{Fe} / \mathrm{H}]$ (upper panel of the figure). We compare our values to the results of the high-quality analysis by Spite et al. (2005). The comparison sample is definitely more metal poor, but we can see the very similar pattern. In the central panel of Fig. 12, [N/Fe] is shown, while in the lower panel $[\mathrm{C}+\mathrm{N} / \mathrm{Fe}]$. The stars in our sample showing an enhanced $\mathrm{A}(\mathrm{N})$ are on average hotter than those in Spite et al. (2005), but this could be explained by looking at the HertzsprungRussell (HR) diagram in Fig. 5, several (probably the majority) of the evolved stars in our sample are AGBs; it is likely that on average they are more evolved than the stars in the sample of Spite et al. (2005), whose average temperature is lower, so that a fraction of them probably still belong to the red giant branch (RGB). 
Table 1. Coordinates, atmospheric parameters, and iron abundance of the programme stars.

\begin{tabular}{|c|c|c|c|c|c|c|c|}
\hline Star & $\begin{array}{c}\text { RA } \\
\text { J2000 }\end{array}$ & $\begin{array}{c}\text { Dec } \\
\text { J2000 }\end{array}$ & $\begin{array}{c}\mathrm{G} \\
\mathrm{mag}\end{array}$ & $\begin{array}{c}T_{\mathrm{eff}} \\
\mathrm{K}\end{array}$ & $\begin{array}{l}\log g \\
{[\operatorname{cgs}]}\end{array}$ & $\begin{array}{c}\xi \\
\mathrm{km} \mathrm{s}^{-1}\end{array}$ & $\begin{array}{c}{[\mathrm{Fe} / \mathrm{H}]} \\
\operatorname{dex}\end{array}$ \\
\hline 01 & & 0.7 & 14.05 & 5269 & - & 1. & 2. \\
\hline & & & & & & & \\
\hline & & 42.41 & & 5280 & & & \\
\hline & & $-49:$ & 4.17 & 5278 & & & 100 \\
\hline & & $-63:$ & & & & & -2.61 \\
\hline & & -57 & & & & & \\
\hline & & $-43:$ & & & & & -1.10 \\
\hline & & $-68:$ & & & & & \\
\hline & & & & & & & \\
\hline & & & & & & & \\
\hline & & 3 & & & & & \\
\hline & & & & & & & \\
\hline & & & & & & & \\
\hline & & 3 & & & & & \\
\hline & & & & & & & \\
\hline & & 6 & & & & & \\
\hline & & & & & & & \\
\hline & & & & & & & \\
\hline & & & & & & & \\
\hline & & & & & & & \\
\hline & & 1 & & 52 & & & \\
\hline & & $-66:$ & & & & & 37 \\
\hline & & & & & & & \\
\hline & & & & & & & \\
\hline & & & & & & & \\
\hline & & & & & & & \\
\hline & & & & & & & \\
\hline & & & & & & & \\
\hline & & & & 61 & & & \\
\hline & & & & & & & \\
\hline & & -43 & & & & & \\
\hline & & -28. & & 54 & & & \\
\hline & & & & & & & \\
\hline & & & & & & & \\
\hline & & & & & & & .12 \\
\hline & & -49 & & & & & $\sim-1$. \\
\hline & & & & & & & \\
\hline & & & & & & & \\
\hline & & & & & & & \\
\hline & & & & & & & \\
\hline & & & & & & & \\
\hline & & & & & & & \\
\hline & & & & 51 & & & \\
\hline & & & & 50 & & & \\
\hline & & & & & & & \\
\hline & & 3 & & 597 & & & \\
\hline & & & & 5205 & & & \\
\hline & & -40 & & 51 & & & 1.0 \\
\hline & & -44 & & 496 & & & 1.1 \\
\hline & & & & 0 & & & 1.4 \\
\hline & & $-38:$ & .26 & 5580 & DU & & -1.36 \\
\hline & & & & 580 & ? & & 1.2 \\
\hline & & & & 908 & 72 & & 1.82 \\
\hline & & & & 5222 & .36 & & -1.04 \\
\hline & & & & 5233 & & & \\
\hline & & & & & & & \\
\hline & & & & & & & \\
\hline & & & & 51 & & & \\
\hline & & & & 50 & & & \\
\hline & & & & & & & \\
\hline & & & & & & & \\
\hline & & & & 518 & & & \\
\hline CHS63 & 00:11:12.94 & $-29: 08: 25.8$ & 14.04 & 5507 & 2.42 & 1.8 & \\
\hline
\end{tabular}

Table 1. continued.

\begin{tabular}{lccccccc}
\hline \hline Star & $\begin{array}{c}\mathrm{RA} \\
\mathrm{J} 2000\end{array}$ & $\begin{array}{c}\text { Dec } \\
\text { J2000 }\end{array}$ & $\begin{array}{c}\mathrm{G} \\
\mathrm{mag}\end{array}$ & $\begin{array}{c}T_{\text {eff }} \\
\mathrm{K}\end{array}$ & $\begin{array}{c}\log g \\
{[\mathrm{cgs}]}\end{array}$ & $\begin{array}{c}\xi \\
\mathrm{kms}^{-1}\end{array}$ & $\begin{array}{c}{[\mathrm{Fe} / \mathrm{H}]} \\
\mathrm{dex}\end{array}$ \\
\hline GHS64 & $22: 19: 10.14$ & $-45: 09: 24.6$ & 14.49 & 5664 & 3.17 & 1.5 & -1.34 \\
GHS65 & $21: 37: 15.25$ & $-41: 26: 44.4$ & 14.31 & 6452 & 3.72 & 1.5 & -2.35 \\
GHS66 & $20: 50: 27.35$ & $-70: 04: 30.9$ & 14.18 & 6800 & 3.94 & 1.5 & -1.02 \\
GHS67 & $20: 24: 19.57$ & $-60: 59: 37.0$ & 14.16 & 5992 & 3.65 & 1.5 & -1.35 \\
GHS68 & $20: 20: 11.70$ & $-76: 00: 57.5$ & 14.09 & 6197 & 3.35 & 1.5 & -1.88 \\
GHS69 & $06: 50: 08.83$ & $-87: 34: 10.5$ & 14.44 & 6665 & 3.81 & 1.5 & -1.94 \\
GHS70 & $15: 01: 14.26$ & $-77: 33: 17.5$ & 14.29 & 6478 & 3.74 & 1.5 & -1.59 \\
GHS71 & $00: 55: 34.23$ & $-58: 02: 58.2$ & 14.21 & 6178 & 3.42 & 1.5 & -2.06 \\
GHS72 & $01: 05: 49.02$ & $-42: 14: 22.2$ & 14.03 & 5671 & 3.17 & 1.5 & -1.49 \\
\hline
\end{tabular}

Notes. The solar reference Fe value is from Caffau et al. (2011b). ${ }^{(a)} \mathrm{RR}$ Lyr stars.

Table 2. Solar abundances.

\begin{tabular}{lcc}
\hline \hline Element & $\mathrm{A}(\mathrm{X})$ & References \\
\hline $\mathrm{C}$ & 8.50 & Caffau et al. (2011b) \\
$\mathrm{N}$ & 7.86 & Caffau et al. (2011b) \\
$\mathrm{Mg}$ & 7.54 & Lodders et al. (2009) \\
$\mathrm{Ca}$ & 6.33 & Lodders et al. (2009) \\
$\mathrm{Ti}$ & 4.90 & Lodders et al. (2009) \\
$\mathrm{Fe}$ & 7.52 & Caffau et al. (2011b) \\
$\mathrm{Ni}$ & 6.23 & Lodders et al. (2009) \\
$\mathrm{Sr}$ & 2.92 & Lodders et al. (2009) \\
$\mathrm{Ba}$ & 2.17 & Lodders et al. (2009) \\
\hline
\end{tabular}

\section{Discussion}

\subsection{Chemical composition}

Due to the extreme kinematics requested in the selection, the stars presented here almost surely belong to the halo, or are unbound stars passing through the Galaxy. We would have expected a large distribution in metallicity (passing-by stars could be of any metallicity), with a preference towards low metallicity, due to the preference we gave to blue objects in the colour selection. Instead, we have a small range in metallicity. Taking into account the complete sample $\langle[\mathrm{Fe} / \mathrm{H}]\rangle=$ $-1.51 \pm 0.42$; but if we restrict to $[\mathrm{Fe} / \mathrm{H}]>-2$, we obtain $\langle[\mathrm{Fe} / \mathrm{H}]\rangle=-1.37 \pm 0.24$, a scatter star-to-star that is comparable to the average line-to-line scatter in the $[\mathrm{Fe} / \mathrm{H}]$ determination of each star. We have a clear peak at $[\mathrm{Fe} / \mathrm{H}] \approx-1.4$, which is higher than the average value derived by Allende Prieto et al. (2014) in their investigation on metal-poor stars (see Fig. 13).

We expected that some of these high-speed stars were objects captured from dwarf galaxies or even stars belonging to disrupted dwarf galaxies, but here we expected, at least for a few cases, stars with low $\alpha$-enhancement because stars in this metallicity range belonging to some dwarf galaxies have this characteristic. One of the explanations for the low $\alpha$-content in the $\mathrm{dSph}$ galaxies is that they are characterised by slow, bursting, or gasping star formation (e.g. Marconi et al. 1994; Font et al. 2006). On the contrary, most (if not all) of the stars in this sample appear $\alpha$-enhanced by the amount expected for metal-poor Galactic stars. For the complete sample we find $\langle[\mathrm{Mg} / \mathrm{Fe}]\rangle=$ $0.42 \pm 0.11$ and $\langle[\mathrm{Ca} / \mathrm{Fe}]\rangle=0.31 \pm 0.10$. The average line-toline scatter in $\mathrm{A}(\mathrm{Mg})$ is 0.14 dex and in $\mathrm{A}(\mathrm{Ca}) 0.21$ dex. Only one star in the sample, GHS66, has the ratios of both $\alpha$-indicators 

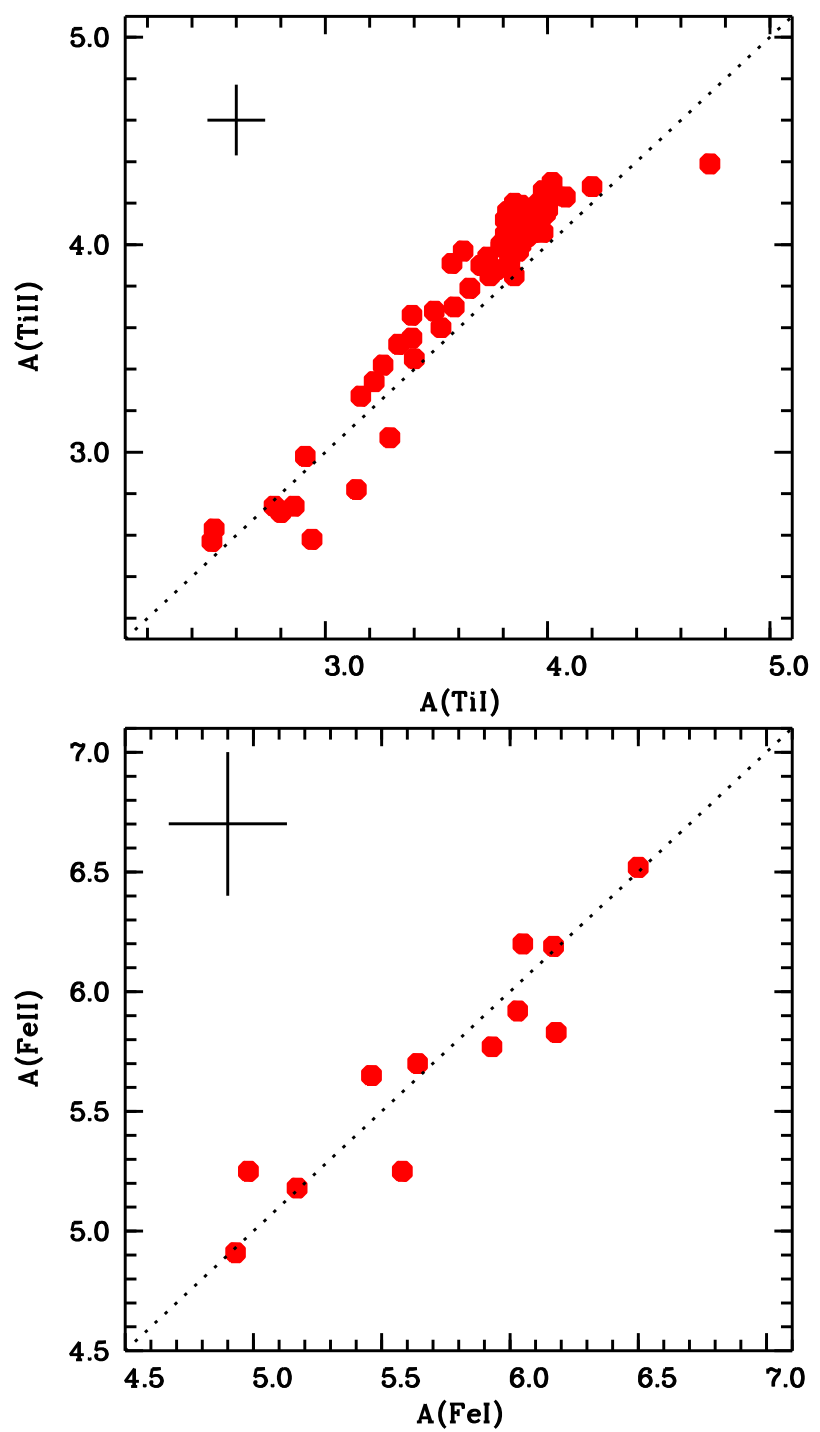

Fig. 7. Comparison of the abundances derived from neutral and ionised elements. To provide an idea of the uncertainties, an average error bar is shown in the plot.

over Fe below $0.2([\mathrm{Mg} / \mathrm{Fe}]=0.18$ and $[\mathrm{Ca} / \mathrm{Fe}]=0.17)$; GHS71 is low in $\mathrm{Ca}([\mathrm{Ca} / \mathrm{Fe}]=0.07)$, but its $\mathrm{Mg}$ is normally enhanced $([\mathrm{Mg} / \mathrm{Fe}]=0.31)$; GHS65 has $[\mathrm{Mg} / \mathrm{Fe}]=0.28[\mathrm{Ca} / \mathrm{Fe}]=0.17$.

Already Haywood et al. (2013) had, in the sample they discussed, a small number of slightly metal-poor $\alpha$-enhanced stars that are young. It is true that the metallicity in their sample is higher $([\mathrm{Fe} / \mathrm{H}] \sim-0.5)$ than the metallicity of our young stars, but they could still belong to the same population. Chiappini et al. (2015) discussed a group of $\alpha$-enhanced stars analysed by CoRoT. These stars with precise age determinations happen to be young and with metallicity from solar to slightly metal poor. According to the authors, these stars could originate near the Galactic bar. We are not sure if our sample of metal-poor young stars is connected to these stars, with good age determinations, studied in the literature.

In Fig. 14 the two $\alpha$-elements analysed here, $\mathrm{Mg}$ and $\mathrm{Ca}$, are compared to the analysis by Nissen \& Schuster (2010). For $\mathrm{Ca}$ we can see no difference in the three populations analysed by Nissen $\&$ Schuster (2010, the low- $\alpha$, accreted population; the high- $\alpha$, halo population; the thick disc population), but for $\mathrm{Mg}$ there is a clear distinction, and from the upper panel of Fig. 14 it

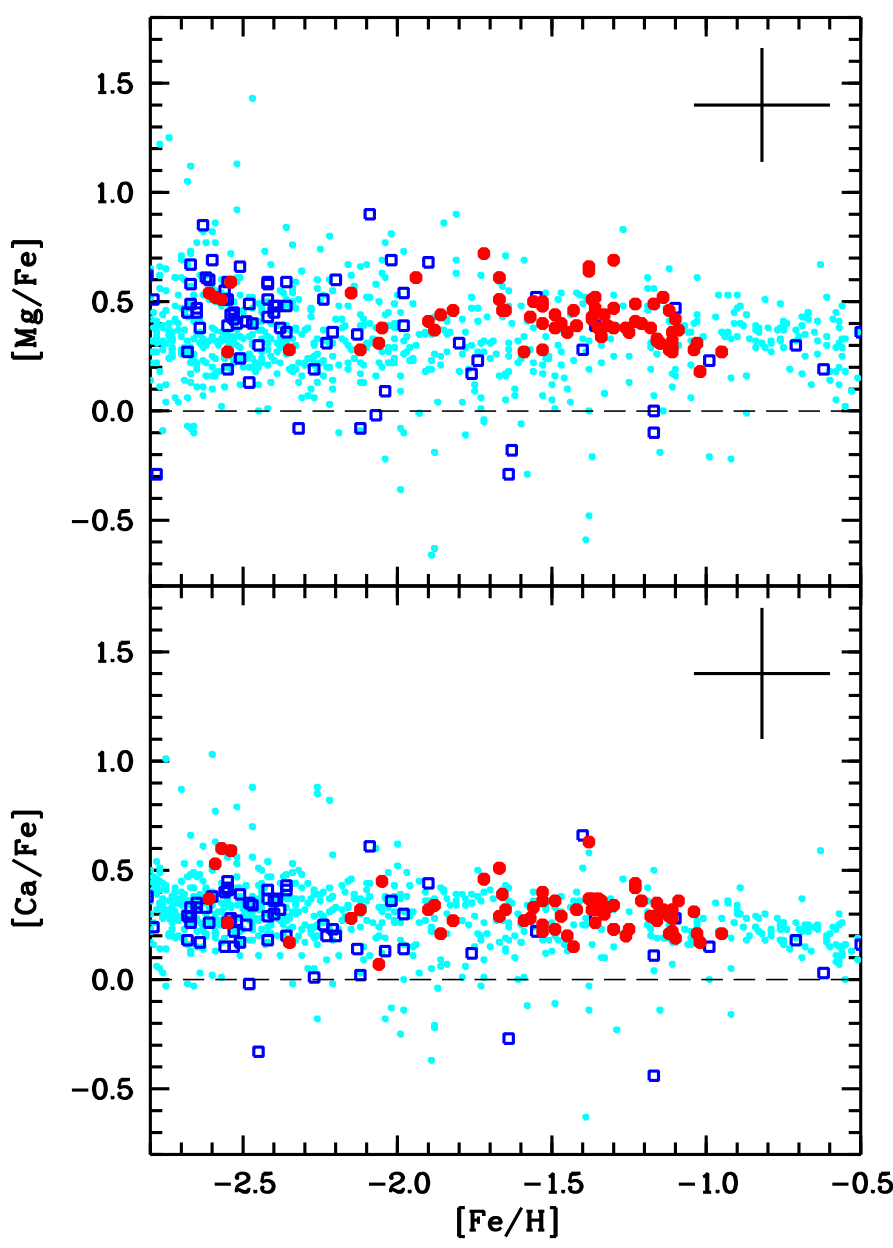

Fig. 8. Two $\alpha$-elements $\mathrm{Mg}$ and $\mathrm{Ca}$ vs. $[\mathrm{Fe} / \mathrm{H}]$ (filled red circles) in comparison with the Caffau et al. (2020) sample (blue squares) and the literature compilation from Frebel (2010, cyan symbols).

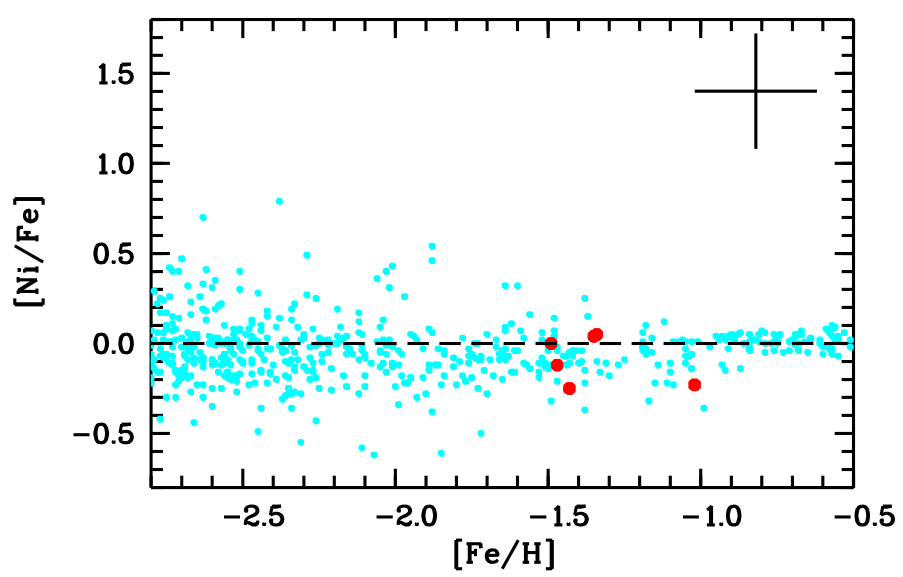

Fig. 9. $[\mathrm{Ni} / \mathrm{Fe}]$ vs. $[\mathrm{Fe} / \mathrm{H}]$ (red filled circles) in comparison with the literature compilation from Frebel (2010) (cyan dots).

is clear that our stars are more consistent with the in situ population by Nissen \& Schuster (2010).

In Fig. $15,[\mathrm{Ca} / \mathrm{Fe}]$ (as a proxy for $[\alpha / \mathrm{Fe}]$ ) as a function of $[\mathrm{Fe} / \mathrm{H}]$ for our stellar sample is compared to literature values from a few local group galaxies. From the figure it is clear that the stars analysed here are compatible with Galactic stars (light grey in the figure), as it was clear from Fig. 8, and with stars belonging to Sgr (black open circles in the figure). If these 


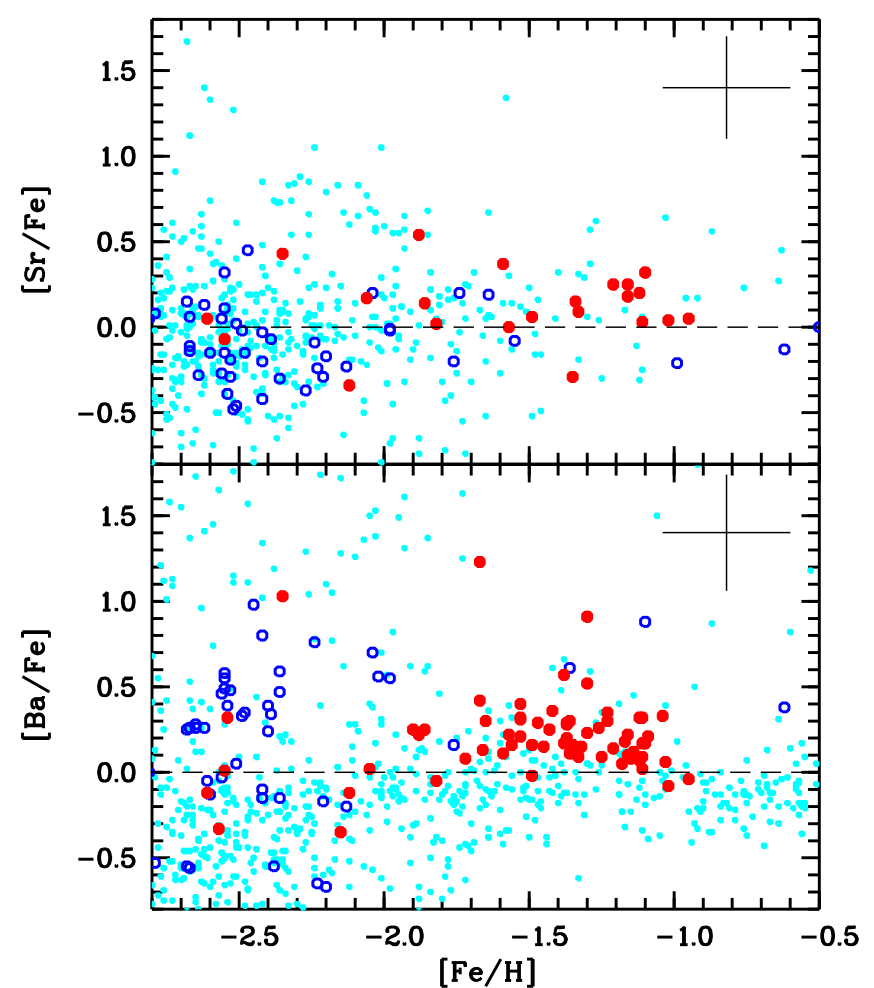

Fig. 10. Heavy elements $[\mathrm{Sr} / \mathrm{Fe}]$ and $[\mathrm{Ba} / \mathrm{Fe}]$ vs. $[\mathrm{Fe} / \mathrm{H}]$ (filled red circles) compared to the Caffau et al. (2020) sample (blue open squares) and the literature compilation from Frebel (2010) (cyan symbols).

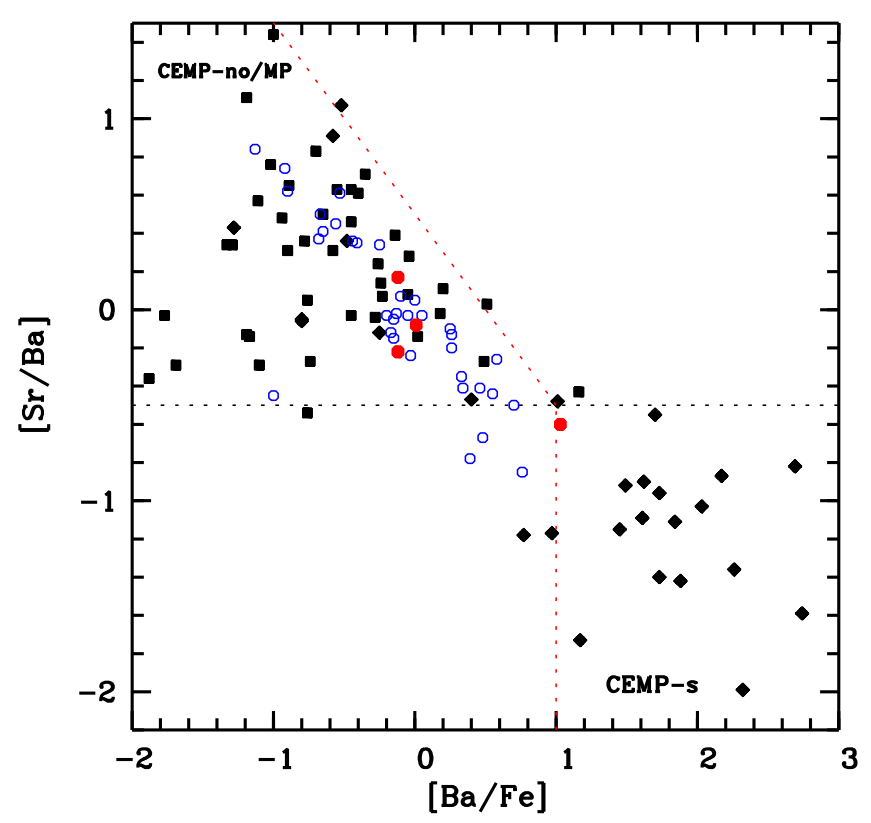

Fig. 11. $[\mathrm{Sr} / \mathrm{Ba}]$ vs. $[\mathrm{Ba} / \mathrm{Fe}]$ for the stars in our sample with $[\mathrm{Fe} / \mathrm{H}]<-2$ (red filled circles) compared to the sample by Caffau et al. (2020, black open symbols) and to metal-poor stars from the literature (blue open symbols).

high-speed stars were formed in dwarf galaxies, then these galaxies should have a star formation history similar to that of Sgr, starting the descent of the $\alpha$-to-iron ratio at a metallicity higher than -1.3 . This suggests fairly massive galaxies, at least like Sgr. Alternatively, we know that globular clusters have very homogeneous Ca-to-Fe ratios ( $\mathrm{Mg}$ may vary because of the presence of

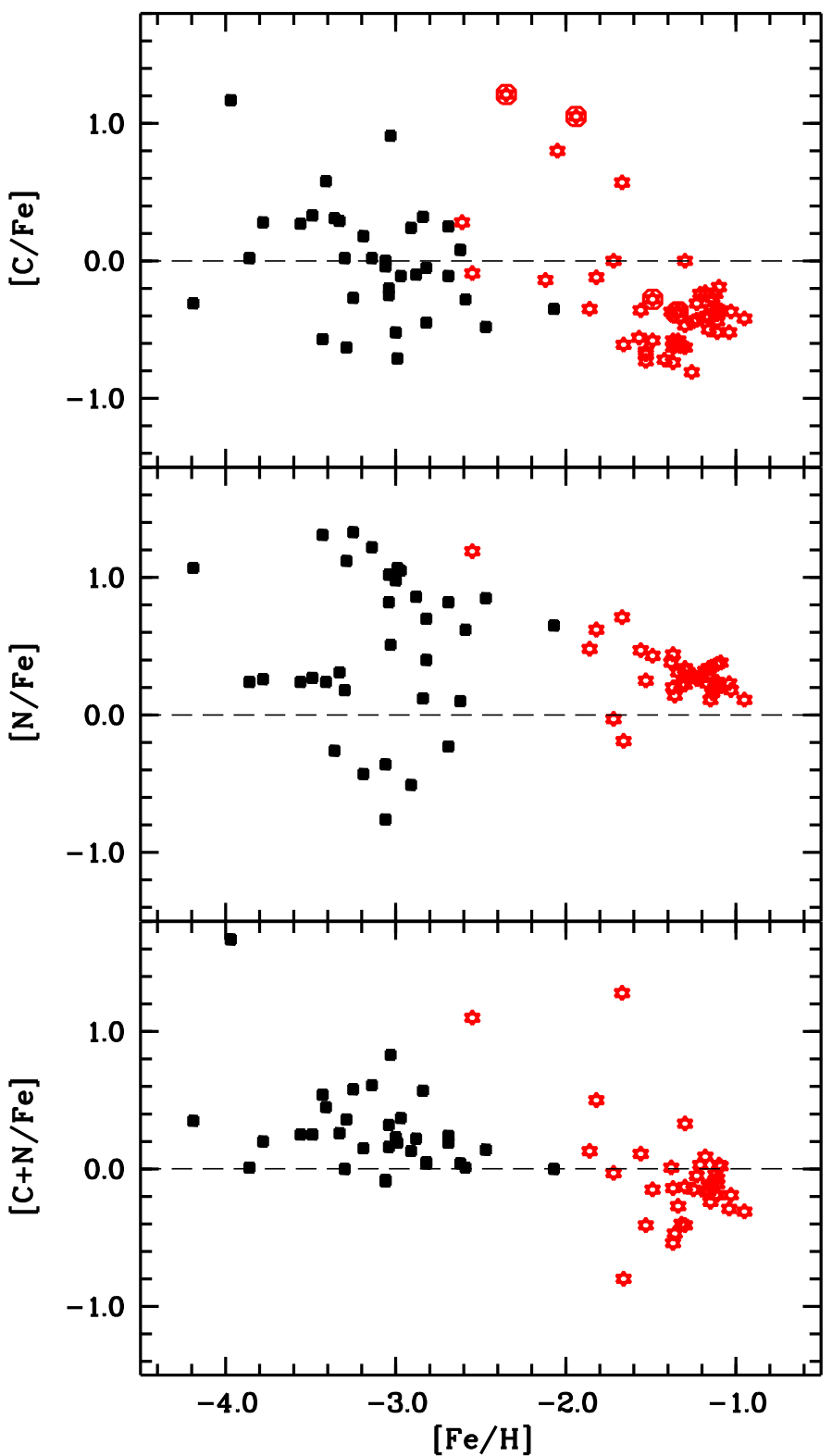

Fig. 12. On a $[\mathrm{Fe} / \mathrm{H}]$ scale, we show for our programme stars (red symbols), $[\mathrm{C} / \mathrm{Fe}]$ (upper panel), $[\mathrm{N} / \mathrm{Fe}]$ (central panel), and $[\mathrm{C}+\mathrm{N} / \mathrm{Fe}]$ (lower panel). The red star symbols surrounded by a circle highlight stars with $\log g>3.0$. The comparison sample shows the very highquality analysis by Spite et al. (2005).

a Mg-Al anti-correlation, initially found by Gratton et al. (2001) and confirmed by many subsequent studies; see Gratton et al. (2004) and Bastian \& Lardo (2018) for reviews on multiple populations in globular clusters), with Ca being enhanced, as in field Milky Way stars. Therefore, our high-speed stars could also have come from globular clusters. High-resolution spectroscopy could shed light on the origin of these stars. If, for instance, they were formed in globular clusters, we would expect to find a Na-O anticorrelation, and perhaps also a $\mathrm{Mg}-\mathrm{Al}$ anti-correlation. At the very least we should be able to find a sizeable dispersion in $\mathrm{Na}$ abundances in any given metallicity bin.

It may be more difficult to find chemical signatures that point to an origin in a dwarf galaxy. For example, in Sgr a top-light initial mass function has been invoked to explain the observed abundances of neutron capture elements (especially for $\mathrm{Eu} / \mathrm{O}$ ) and of $\mathrm{Cu}$ (McWilliam et al. 2013). However, most 


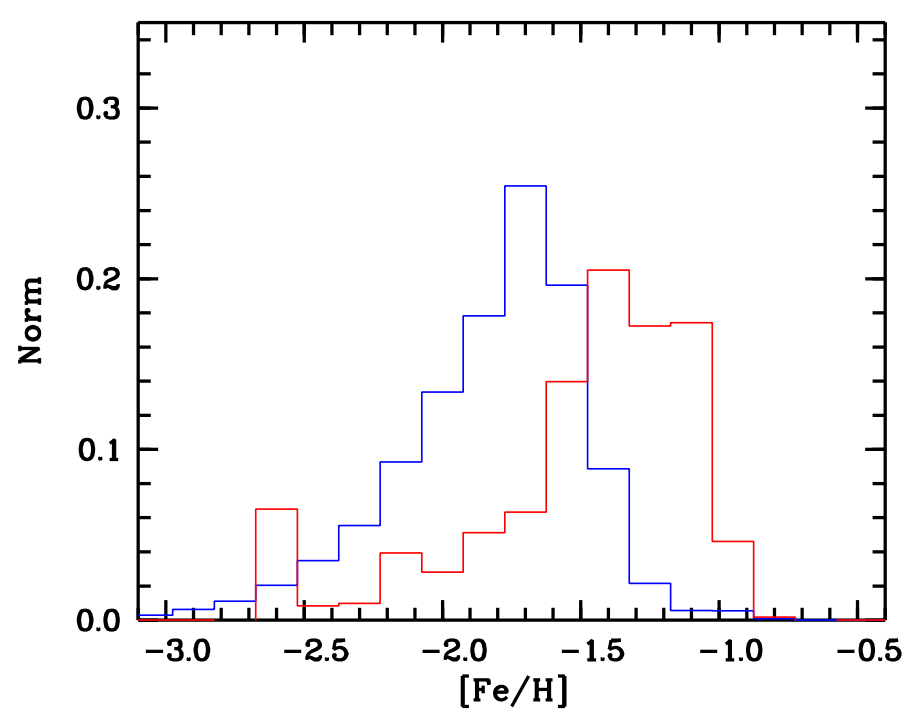

Fig. 13. Normalised $[\mathrm{Fe} / \mathrm{H}]$ distribution (solid red) compared to the result from Allende Prieto et al. (2014) (solid blue).

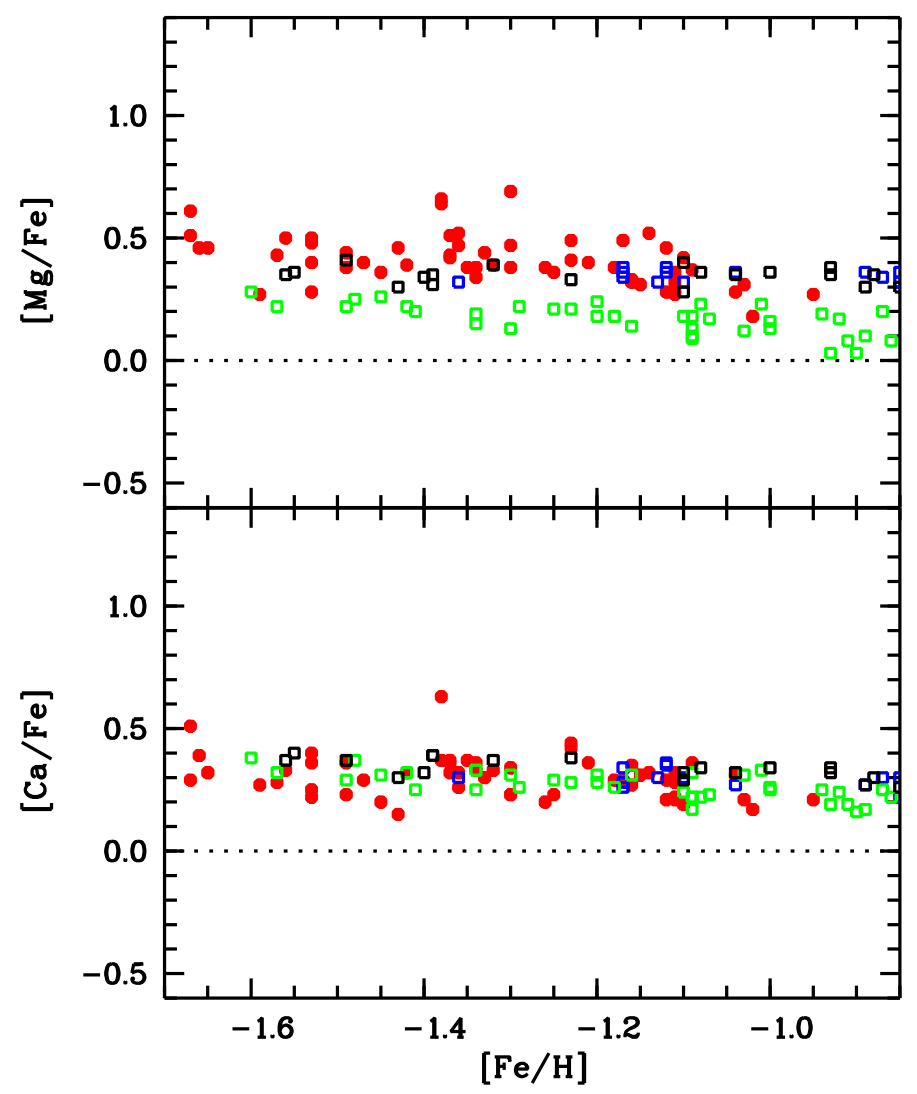

Fig. 14. Abundances of $\alpha$-elements vs. [Fe/H]: solid red symbols are the stars here analysed, compared with the data by Nissen \& Schuster (2010) as open squares, green the low- $\alpha$, black the high- $\alpha$ and blue thick-disc stars.

of the signal comes from stars with $[\mathrm{Fe} / \mathrm{H}]>-1.0$; for the metalpoor population the abundance ratios are the same as those of Milky Way stars (Hansen et al. 2018). Roederer et al. (2018) have claimed that stars with enhanced neutron capture elements formed in the $r$-process ( $r$-enhanced for short) have been formed in dwarf galaxies and accreted to the Milky Way; however, Di Matteo et al. (2020) refuted this claim on the grounds that $r$-enhanced stars are kinematically indistinguishable from

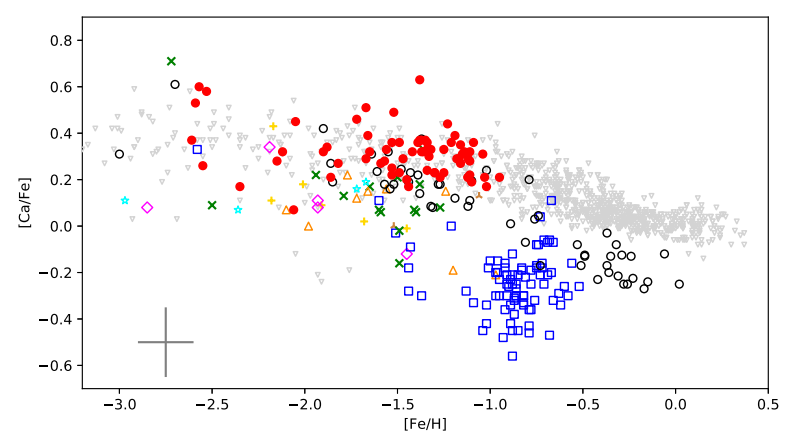

Fig. 15. $[\mathrm{Ca} / \mathrm{Fe}]$ as a proxy for the $\alpha$-enhancement vs. $[\mathrm{Fe} / \mathrm{H}]$ for our sample of stars (red filled circles) compared to data of the Milky Way and the dwarf local group galaxies from the literature. Shown are open grey triangles, MW stars (Venn et al. 2004; Reddy et al. 2006); black open circles, Sgr dSph main body stars (Monaco et al. 2005; Sbordone et al. 2007, 2015, and in prep.; Hansen et al. 2018; open blue squares, dSph (Venn et al. 2004; Letarte 2007); green "x" signs, Car dSph (Venn et al. 2004; Koch et al. 2008); orange open triangles, Scl dSph (Venn et al. 2004; Geisler et al. 2005). All remaining symbols are from Venn et al. (2004): open magenta diamonds, Sex dSph; brown triangles Leo dSph; yellow plus signs, Umi dSph; open cyan stars, Dra dSph.

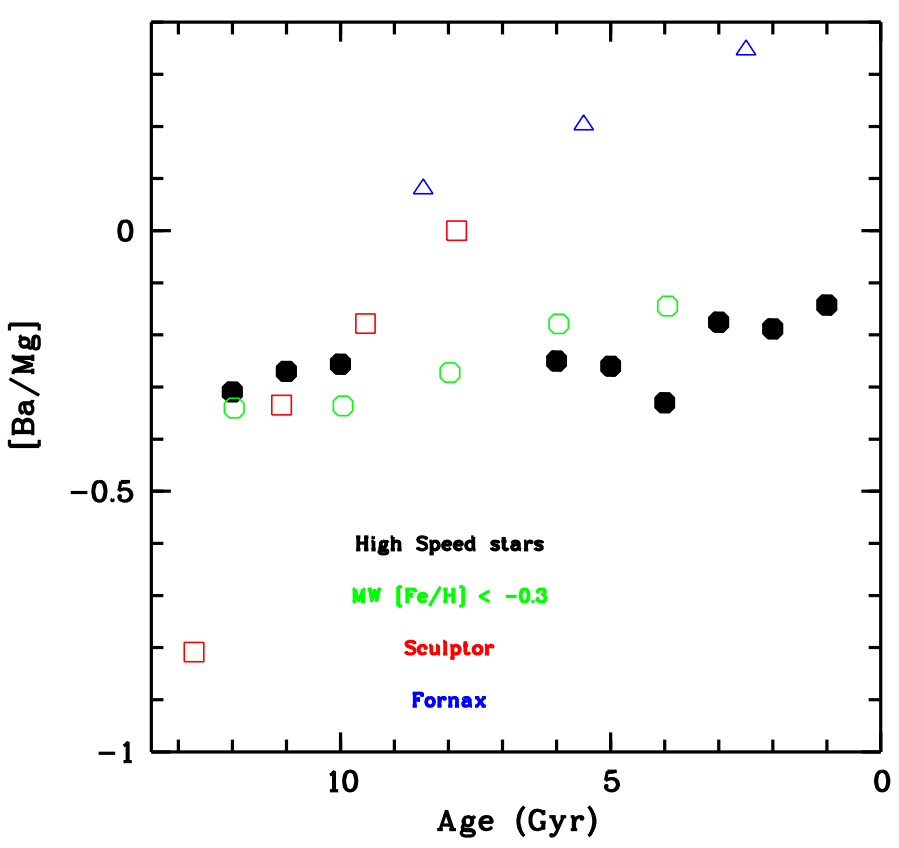

Fig. 16. $[\mathrm{Ba} / \mathrm{Mg}]$ vs. age for Sculptor, Fornax, and Milky Way stars with $[\mathrm{Fe} / \mathrm{H}]<-0.3$, from Skúladóttir et al. (2019), compared with our highspeed stars. The latter seem very consistent with MW stars, but not with Sculptor or Fornax.

$r$-normal stars. Skúladóttir et al. (2019) have shown that the growth of $[\mathrm{Y} / \mathrm{Mg}]$ and $[\mathrm{Ba} / \mathrm{Mg}]$ as a function of age are clearly different among different galaxies and among samples of Milky Way stars in different metallicity bins. In Fig. 16 our [Ba/Mg] results for bins in ages are compared to the curves for different galaxies by Skúladóttir et al. (2019). Clearly our values are in better agreement with the Milky Way results than with any of the other dwarf galaxies shown in the plot.

For $[\mathrm{Ba} / \mathrm{Fe}]$ the comparison of our sample with the sample analysed by Nissen \& Schuster (2011), shown in Fig. 17, also suggests that our sample is compatible with the Milky Way and thus not accreted. The comparison in Fig. 18 also shows an 


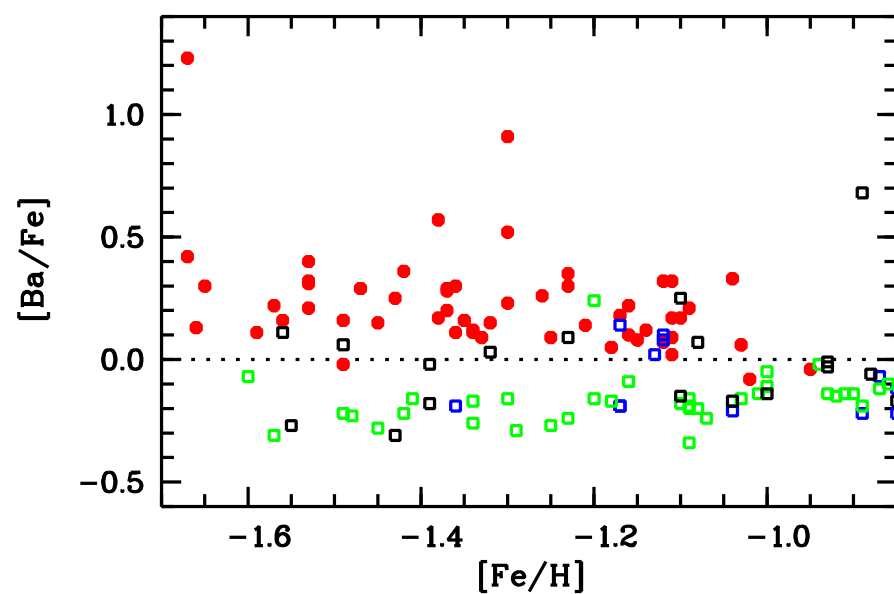

Fig. 17. $[\mathrm{Ba} / \mathrm{Fe}]$ vs. $[\mathrm{Fe} / \mathrm{H}]$ : solid red symbols are the stars analysed here, compared with the data by Nissen \& Schuster (2011) as open squares, low- $\alpha$ in green, high- $\alpha$ in black, and thick-disc stars in blue.

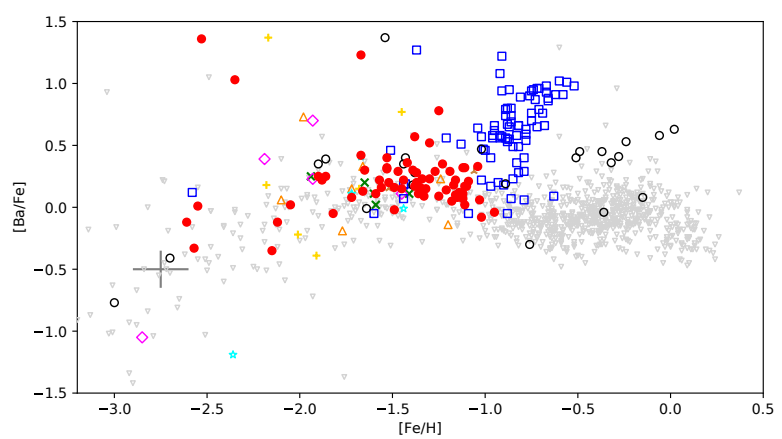

Fig. 18. $[\mathrm{Ba} / \mathrm{Fe}]$ vs. $[\mathrm{Fe} / \mathrm{H}]$, comparison with the literature values. Symbols are as in Fig. 15.

agreement with the Galactic sample more than with a sample from dwarf spheroidal galaxies.

Overall, we expected to find a large chemical variation from star to star in the sample; instead, we find a homogeneous sample from this low-resolution analysis, with no chemical-peculiar star except two CEMP stars (GHS65 and GHS69). Could these stars, at least the ones with $[\mathrm{Fe} / \mathrm{H}]>-2$, belong to a disrupted globular cluster? Or could they all come from a disrupted small galaxy, similar to Sgr?

\subsection{Variability}

The two known RR Lyr stars (GHS29, GHS46) lie in the instability strip of the fundamental mode according to Bono \& Stellingwerf (1994). In Fig. 19 they are compared to the other star in the sample. Four more stars lie in the instability strip: GHS24, GHS36, GHS42, GHS56. GHS36 has a strange spectrum, with broad H-lines. For the other three we have no special remarks.

\subsection{Ages}

The subgiant stars $(\log g>3)$ lie in an HR part of the diagram where, once the metallicity is known, the age of the star can be inferred by using an isochrone (see Fig. 6). We are absolutely aware of the uncertainty of this method, but it can give us a rough estimation of the age or at least tell us if the ages of these metal-

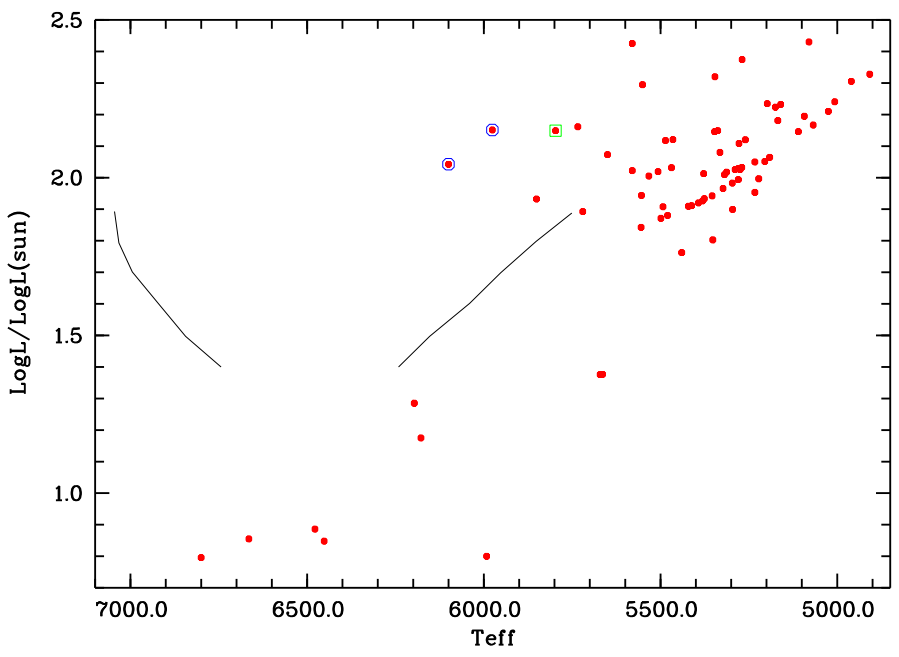

Fig. 19. Hertzsprung-Russell diagram. The two known RR Lyr stars are highlighted by blue circles. The suspected RR Lyr star or binary, GHS36, by a green square. The two black curves are the blue edge and the red edge of the fundamental mode of pulsation of RR Lyr according to Bono \& Stellingwerf (1994).

poor stars are compatible with ages of the order of or larger than about $10 \mathrm{Gyr}$, as expected for metal-poor stars. On the contrary, all these stars are compatible with ages of $8 \mathrm{Gyr}$ or younger. One problem could be the reddening that shift the stars to younger positions in the HR diagram. If no reddening is applied, some stars will surely take places in the colour-colour diagram close to an isochrone of a more appropriate older age, but not all. We conclude that the reddening can play a role in making some stars to appear younger, but not all. We could assume that these metalpoor apparently young stars are blue stragglers. Assuming that this is the case, by selecting high-speed stars that are bluer than the -2.0 metallicity isochrone, we end up with all the subgiant stars being blue stragglers. Is high speed a result of the formation of a blue straggler?

For the more evolved $(\log g<3)$ stars things are more complicated. There are 19 stars for which we use isochrones of 10 or $12 \mathrm{Gyr}$ to derive the parameters, but for the other 44 stars younger ages are needed to fit well the photometry of the stars on the isochrones. What is evident is that the isochrones of different ages are close enough to make it difficult to rule out that most of the stars are in fact about 10-12 Gyr old; even so, Fig. 6 convinces us that several of these stars are in fact younger than 12 Gyr. Without a doubt, some of the stars are in the HR diagram in places where no 10 or $12 \mathrm{Gyr}$ old isochrone can be found. In addition, if our selection results in so many young subgiant stars that we could easily classify as blue stragglers, why is it not possible that some of these more evolved stars are the evolution of these blue stragglers? Or, even if the subgiant populations is in fact composed of young stars, why should it not also be the case for the giant stars?

The point is then to find an explanation that justifies the high speed and young ages in this sample of stars.

\subsection{Kinematics}

Some stars have a maximum distance from the centre of the Galaxy that is much larger than its estimated radius. Twentythree stars reach distances larger than $100 \mathrm{kpc}$ from the centre of the Galaxy. In particular, three stars (GHS32, GHS56, and GHS57) reach more than $300 \mathrm{kpc}$ from the centre of the Milky 
Way. Clearly, for these stars in particular, to be sure that they are really bound and in order to derive their orbits and orbital parameters, we should take into account all the dwarf local galaxies in the potential, but probably also the Magellanic clouds and perhaps even Andromeda.

Clearly the sample is small, but we still tried to see if we could identify streams. We first checked whether our stars are consistent with the streams in Malhan et al. (2018), but we could find none. In a few cases the coordinates were consistent with known streams, but our stars are much further away. We looked for a subsample of stars close in proper motion, and we found two of them. These stars are probably close in proper motion just by chance; in fact, we did not find in the Gaia DR2 catalogue other stars that could form a stream consistent with these stars.

Five stars in the sample have unbound orbits (GHS22, GHS33, GHS37, GHS58, GHS64) at $1 \sigma$ significance, due mainly to the uncertainty on the parallax. When considering the formal error on the total speed, we find that for all five stars it still exceeds the Galactic escape speed (taken to be $528_{-25}^{+24} \mathrm{~km} \mathrm{~s}^{-1}$; Deason et al. 2019) even after subtracting a $1 \sigma$ error from it, but not if we subtract $2 \sigma$. The same holds true by taking into account the variation of the escape velocities with the distance from the Galactic centre (see Monari et al. 2018).

\subsection{Possible origins of our sample high-speed stars}

The selection of our sample, as described in Sect. 2, is heavily kinematically biased in colour and luminosity. In this respect we have to keep in mind that we are selecting a very rare population. Our colour-magnitude selection of the already rare high-speed sample selected only $4 \%$ of the stars. It is therefore legitimate to also consider the possibility that our stars trace several very rare events. If this were the case we would expect our stars to be very diverse in terms of chemical composition.

Cross-matching GaiaDR2 with LAMOST allowed us to identify 68 high-speed late-type stars for which the LAMOST pipeline provides atmospheric parameters and chemical abundances (Du et al. 2018a,b, 2019). None of these stars is also in our sample. Although Du et al. (2019) comment that "Most of the high velocity stars are metal-poor and $\alpha$-enhanced", inspection of Fig. 4 of Du et al. (2018a) and Fig. 2 of Du et al. (2018b) shows that there is a sizeable spread in $\alpha$-to iron ratios, with several stars with low ratios and also a considerable spread in metallicity. This is not the case in our sample. The precision in abundances is probably the same for the LAMOST data and ours; we use a slightly higher resolving power, but the LAMOST spectra have a much larger spectral coverage. It should be noted that the selection of the two samples of stars is different: Du and collaborators were interested in detecting unbound stars; we were aiming at the metal-poor population of high-speed stars. Another difference is that we performed spectroscopic observations following our hybrid kinematical and colour-magnitude selection, while $\mathrm{Du}$ and collaborators just picked the stars that were independently targeted by LAMOST without any kinematical selection.

The availability of Gaia DR2 triggered many works searching for unbound stars, and many of these studies can be found in the works of $\mathrm{Du}$ and collaborators; we mention here only the work of Bromley et al. (2018). Star Gaia DR2 5846998984508676352 is in common between Bromley et al. (2018) and our sample of high-velocity stars, but we did not observe it because it was too cool and faint for our selection. Although they did not select preferentially brighter and bluer stars, as we did, it is nicely illustrated in their Fig. 7 that the unbound stars are the most luminous for any given colour. We interpret this as an effect of the Malmquist bias, as for our sample. This implies that the speed selections will always be biased towards brighter younger and metal-poor stars. However there is no obvious reason why the metallicity range should be limited.

The results of our analysis show that, on the one hand the metallicity range of our sample is very limited and that the abundance ratios are also very tightly clustered. While our preference for blue stars does explain the fact that all of the sample is metal poor, we expected a larger metallicity spread; in particular, we expected to observe at least one extremely metal-poor star with $[\mathrm{Fe} / \mathrm{H}]<-3.0$, as found by Du et al. (2018a). In the same spirit, we expected a large spread in $\alpha$-to-iron ratios and barium-to-iron ratios, which is hardly the case.

Unless we admit that these stars were born with such extreme velocities we must assume that they were accelerated. Let us begin by examining the possible causes for acceleration:

1. Merging of an external galaxy with the Milky Way;

2. Interaction with black hole in the Galactic centre (Hills 1988);

3. Origin in an external galaxy (e.g. Andromeda, see Sherwin et al. 2008) and ejection through interaction with its central black hole;

4. Star in a binary system with a massive primary that ended its life as a SN and gave a "kick" to the secondary;

5. Star in a hierarchical triple system, with a massive primary that ended its life as a supernova (SN) and gave a kick to the outer couple that remained bound;

6. Origin in a globular cluster and ejection through gravitational interaction in an intermediate mass black hole at the centre of the cluster (see e.g. Fragione \& Gualandris 2019, and references therein).

Scenario 1 requires that the merging galaxy be rather massive (but still less than the MW) and with a star formation history that implies a constant $\alpha$-to-iron ratio in the metallicity range probed by the present sample. However, in such a case, according to the simulations of Jean-Baptiste et al. (2017), in the phase-space domain explored we should find both MW stars and stars formed in the accreted galaxy, although the latter are favoured among high kinetic energy stars. Even if in the presently explored metallicity regime the two populations were indistinguishable in terms of $\alpha$-to-iron ratios, as we move to a more metal-rich regime we expect that the two populations should split, the accreted galaxy showing lower $\alpha$-to-iron ratios. Figure 1 shows clearly that among these high-speed stars selected (black dots) the majority lie to the red of the metal-poor isochrone $([\mathrm{M} / \mathrm{H}]=-2.0)$, so they are indeed more metal-rich than the observed stars. It would therefore be extremely interesting to measure $\alpha$-to-iron ratios in the more metal-rich high-speed stars to test this possible interpretation.

Scenarios 2, 3, 4, and 5 are all plausible, and not mutually exclusive. In particular, there are four targets whose minimum distance from the Galactic Centre is less than $0.5 \mathrm{kpc}$, making an origin in this environment (scenario 2) plausible. The star GHS64 is compatible with scenario 3 (see Sect. 5.4), yet there is no reason why a multiplicity of these rare events should end up with such a high chemical homogeneity, as observed. Scenario 5 in particular would also be able to explain the presence of evolved blue stragglers: after the kick due to the SN explosion, the remaining binary undergoes merging and becomes a blue straggler.

Scenario 6 is the most appealing. The weak point is the fact that no conclusive evidence yet exists for the presence 
of intermediate black holes at the centres of globular clusters, although there are strong theoretical reasons to suggest that this is the case (Greene et al. 2019). There are two reasons that make this scenario appealing: in the first place, the metallicity distribution and $\alpha$-to-iron ratios of our sample are consistent with their origin in Galactic globular clusters (GGC); in the second place, this would explain the presence of blue stragglers, which we know populate GGC. This idea can be put directly to test in two ways. We know that GGC are characterised by multiple stellar populations that give rise to several correlations, and anti-correlations among abundance ratios, the most prominent being the $\mathrm{Na}-\mathrm{O}$ anti-correlation, as mentioned in Sect. 5.1. Unfortunately our data resolution is too low to provide reliable $\mathrm{Na}$ abundances; the only $\mathrm{Na}$ I lines detected are the D-doublet, and at our resolution it is difficult to disentangle the stellar absorption from the interstellar absorption and the atmospheric emission, even for the stars with the highest radial speeds. Thus, the first test would be to observe a sizeable fraction of these stars at high resolution and see if a Na-O anti-correlation becomes apparent, or at least a large spread in $\mathrm{Na}$ abundances. In absence of this we could easily rule out the origin in GGC.

The second test concerns the subgiant and turn-off stars that appear to be younger than $10 \mathrm{Gyr}$. If they are blue stragglers then they should show no measurable Li if they were formed from the coalescence of the components of a binary system, or Li should be depleted $(\mathrm{A}(\mathrm{Li}) \sim 1)$ if the blue straggler was formed by collision (Glebbeek et al. 2010). If the stars are not blue stragglers, then their lithium abundance should lie on the Spite plateau at $\mathrm{A}(\mathrm{Li}) \sim 2.2$ (Spite \& Spite 1982; Sbordone et al. 2010).

We can see that the metallicity of this sample of stars peaks at about -1.4 (see Fig. 13), in surprising agreement with the peak of the Gaia-Sausage-Enceladus sample (see Fig. 19 in Di Matteo et al. 2019). This intriguing fact deserves further investigation.

\section{Conclusions}

We presented here the chemodynamical investigation of a sample of 72 stars, selected from the Gaia DR2 catalogue for their high transversal velocity and observed with FORS at the VLT. Our main conclusions, discussed in detail in the previous section, are summarised below:

- The chemical pattern of these stars is extremely homogeneous. Although the stars in the sample analysed here suffer from a strong selection bias, we believe that the relatively narrow range in metallicity, from metal poor to very metal poor, is too extreme to be explained by our selection bias. Even if the analysis is based on low-resolution spectra, implying a larger uncertainty, the homogeneity in their abundances is comparable to the high-quality sample analysed by Spite et al. (2005).

- The subgiant stars in the sample all appear to be younger than expected at this metallicity range, being of $8 \mathrm{Gyr}$ of age or younger. With this selection we could have picked up mainly (or only) blue stragglers.

- For the giant stars, a minority $(30 \%, 19 / 63)$ are as old as expected, while others appear generally much younger than expected $(70 \%, 44 / 63$ with an age of 8 Gyr or younger, up to 1 Gyr). They could be evolved blue stragglers.

- Most or all of these stars show a high $[\alpha / \mathrm{Fe}]$, compatible with the MW population or (in this metallicity regime) the population of massive satellites like Sgr.
- Some stars have extreme orbits. As expected, all the other stars show halo orbits. Few stars reach such large distances from the centre of the Galaxy to cast doubts on their boundness.

- We identified several acceleration mechanism (see Sect. 5.5) of which the one able to explain the chemical homogeneity is an origin of these stars in GGCs. We suggested observations to probe this scenario.

- We were able to identify two small subsamples of stars close in proper-motion, but we could not find other stars in the Gaia DR2 catalogue belonging to these hypothetical streams.

Acknowledgements. We wish to thank the anonymous referee for the help provided in improving the paper. Based on observations collected at the European Southern Observatory under ESO programme 0104.D-0259. We gratefully acknowledge support from the French National Research Agency (ANR) funded project "Pristine" (ANR-18-CE31-0017). EC and PB are thankful to ESO Santiago for hosting them during the analysis of this sample of stars. This work has made use of data from the European Space Agency (ESA) mission Gaia (https://www.cosmos.esa.int/gaia), processed by the Gaia Data Processing and Analysis Consortium (DPAC, https://www.cosmos.esa.int/ web/gaia/dpac/consortium). Funding for the DPAC has been provided by national institutions, in particular the institutions participating in the Gaia Multilateral Agreement. This research has made use of the SIMBAD database, operated at CDS, Strasbourg, France.

\section{References}

Allende Prieto, C., Fernández-Alvar, E., Schlesinger, K. J., et al. 2014, A\&A, 568, A7

Appenzeller, I., Fricke, K., Fürtig, W., et al. 1998, Messenger, 94, 1

Arenou, F., Luri, X., Babusiaux, C., et al. 2018, A\&A, 616, A17

Bastian, N., \& Lardo, C. 2018, ARA\&A, 56, 83

Belokurov, V., Erkal, D., Evans, N. W., et al. 2018, MNRAS, 478, 611

Boyce, E. H. 1943, Harvard Coll. Obs. Bull., 917, 1

Bonifacio, P., Spite, M., Cayrel, R., et al. 2009, A\&A, 501, 519

Bono, G., \& Stellingwerf, R. F. 1994, ApJS, 93, 233

Bovy, J. 2015, ApJS, 216, 29

Bressan, A., Marigo, P., Girardi, L., et al. 2012, MNRAS, 427, 127

Bromley, B. C., Kenyon, S. J., Brown, W. R., et al. 2018, ApJ, 868, 25

Bromm, V., \& Larson, R. B. 2004, ARA\&A, 42, 79

Bromm, V., Yoshida, N., Hernquist, L., et al. 2009, Nature, 459, 49

Caffau, E. 2018, "Turn-Off Primordial Stars" invited talk at "The Metal-poor Galaxy" (Tegernsee, Germany: Schloss Ringberg)

Caffau, E., Bonifacio, P., François, P., et al. 2011a, Nature, 477, 67

Caffau, E., Ludwig, H.-G., Steffen, M., Freytag, B., \& Bonifacio, P. 2011 b, Sol. Phys., 268, 255

Caffau, E., Bonifacio, P., Sbordone, L., et al. 2013, A\&A, 560, A71

Caffau, E., Bonifacio, P., Sbordone, L., et al. 2020, MNRAS, 493, 4677

Cayrel, R., Depagne, E., Spite, M., et al. 2004, A\&A, 416, 1117

Chamberlain, J. W., \& Aller, L. H. 1951, ApJ, 114, 52

Chiappini, C., Anders, F., Rodrigues, T. S., et al. 2015, A\&A, 576, L12

Deason, A. J., Fattahi, A., Belokurov, V., et al. 2019, MNRAS, 485, 3514

Dehnen, W., \& Binney, J. 1998, MNRAS, 294, 429

Demers, S., \& Irwin, M. J. 1991, A\&AS, 91, 171

Di Matteo, P., Haywood, M., Lehnert, M. D., et al. 2019, A\&A, 632, A4

Di Matteo, P., Spite, M., Haywood, M., et al. 2020, A\&A, 636, A115

Du, C., Li, H., Liu, S., et al. 2018a, ApJ, 863, 87

Du, C., Li, H., Newberg, H. J., et al. 2018b, ApJ, 869, L31

Du, C., Li, H., Yan, Y., et al. 2019, ApJS, 244, 4

Eggen, O. J., Lynden-Bell, D., \& Sandage, A. R. 1962, ApJ, 136, 748

Font, A. S., Johnston, K. V., Bullock, J. S., et al. 2006, ApJ, 638, 585

Fragione, G., \& Gualandris, A. 2019, MNRAS, 489, 4543

François, P., Depagne, E., Hill, V., et al. 2007, A\&A, 476, 935

Frebel, A. 2010, Astron. Nachr., 331, 474

Gaia Collaboration (Prusti, T., et al.) 2016, A\&A, 595, A1

Gaia Collaboration (Brown, A. G. A., et al.) 2018, A\&A, 616, A1

Gallart, C., Bernard, E. J., Brook, C. B., et al. 2019, Nat. Astron., 3, 932

Gavrilchenko, T., Klein, C. R., Bloom, J. S., et al. 2014, MNRAS, 441, 715

Geisler, D., Smith, V. V., Wallerstein, G., et al. 2005, AJ, 129, 1428

Giclas, H. L., Burnham, R., \& Thomas, N. G. 1971, Flagstaff (Arizona: Lowell Observatory)

Glebbeek, E., Sills, A., Hu, H., et al. 2010, AIP Conf. Proc., 1314, 113

Gratton, R. G., Bonifacio, P., Bragaglia, A., et al. 2001, A\&A, 369, 87 
Gratton, R., Sneden, C., \& Carretta, E. 2004, ARA\&A, 42, 385

Greene, J. E., Strader, J., \& Ho, L. C. 2019, ARA\&A, submitted [arXiv:1911.09678]

Greif, T. H., Springel, V., White, S. D. M., et al. 2011, ApJ, 737, 75

Hansen, C. J., El-Souri, M., Monaco, L., et al. 2018, ApJ, 855, 83

Haywood, M., Di Matteo, P., Lehnert, M. D., et al. 2013, A\&A, 560, A109

Haywood, M., Di Matteo, P., Lehnert, M. D., et al. 2018, ApJ, 863, 113

Helmi, A., Babusiaux, C., Koppelman, H. H., et al. 2018, Nature, 563, 85

Hills, J. G. 1988, Nature, 331, 687

Jean-Baptiste, I., Di Matteo, P., Haywood, M., et al. 2017, A\&A, 604, A106

Kervella, P., Arenou, F., Mignard, F., et al. 2019, A\&A, 623, A72

Koch, A., Grebel, E. K., Gilmore, G. F., et al. 2008, AJ, 135, 1580

Kruijssen, J. M. D., Pfeffer, J. L., Reina-Campos, M., et al. 2019, MNRAS, 486, 3180

Kukarkin, B. V., Kholopov, P. N., Pskovsky, Y. P., et al. 1971, General Catalogue of Variable Stars

Kurucz, R. L. 2005, Mem. Soc. Astron. It. Supp., 8, 14

Lee, S.-G. 1984, AJ, 89, 702

Letarte, B. 2007, Ph.D. Thesis, Chemical Analysis of the Fornax Dwarf Galaxy, University of Groningen, the Netherlands

Lindegren, L., Hernández, J., Bombrun, A., et al. 2018, A\&A, 616, A2

Lodders, K., Palme, H., \& Gail, H.P. 2009, Landolt Börnstein, 712,

Lopez, C. E., \& Girard, T. M. 1990, PASP, 102, 1018

Ludwig, H.-G., Bonifacio, P., Caffau, E., et al. 2008, Phys. Scr. Vol. T, 133, 014037

Mackereth, J. T., \& Bovy, J. 2020, MNRAS, 492, 3631

Malhan, K., Ibata, R. A., \& Martin, N. F. 2018, MNRAS, 481, 3442

Malmquist, K. G. 1925, Meddelanden fran Lunds Astronomiska Observatorium Serie, I(106),

Marconi, G., Matteucci, F., \& Tosi, M. 1994, MNRAS, 270, 35

Marigo, P., Girardi, L., Bressan, A., et al. 2017, ApJ, 835, 77

Mashonkina, L. I., Sitnova, T. N., \& Pakhomov, Y. V. 2016, Astron. Lett., 42, 606

McMillan, P. J. 2017, MNRAS, 465, 76 (M17)

McWilliam, A., \& Smecker-Hane, T. A. 2005, Cosmic Abundances as Records of Stellar Evolution and Nucleosynthesis, 221

McWilliam, A., Wallerstein, G., \& Mottini, M. 2013, ApJ, 778, 149

Meléndez, J., Placco, V. M., Tucci-Maia, M., et al. 2016, A\&A, 585, L5

Monaco, L., Bellazzini, M., Bonifacio, P., et al. 2005, A\&A, 441, 141
Monari, G., Famaey, B., Carrillo, I., et al. 2018, A\&A, 616, L9

Myeong, G. C., Vasiliev, E., Iorio, G., et al. 2019, MNRAS, 488, 1235

Nissen, P. E., \& Schuster, W. J. 2010, A\&A, 511, L10

Nissen, P. E., \& Schuster, W. J. 2011, A\&A, 530, A15

Penoyre, Z., Belokurov, V., Evans, N. W., Everall, A., \& Koposov, S. E. 2020, MNRAS, 495, 321

Platais, I., Girard, T. M., Vieira, K., et al. 2011, MNRAS, 413, 1024

Reddy, B. E., Lambert, D. L., \& Allende Prieto, C. 2006, MNRAS, 367, 1329

Reina-Campos, M., Hughes, M. E., Kruijssen, J. M. D., et al. 2020, MNRAS, 493, 3422

Roederer, I. U., Hattori, K., \& Valluri, M. 2018, AJ, 156, 179

Roman, N. G. 1950, ApJ, 112, 554

Roman, N. G. 1955, ApJS, 2, 195

Sbordone, L., Bonifacio, P., Castelli, F., \& Kurucz, R. L. 2004, Mem. Soc. Astron. It. Supp., 5, 93

Sbordone, L., Bonifacio, P., Giuffrida, G., et al. 2007, Galaxy Evolution Across the Hubble Time, 330

Sbordone, L., Bonifacio, P., Caffau, E., et al. 2010, A\&A, 522, A26

Sbordone, L., Caffau, E., Bonifacio, P., \& Duffau, S. 2014, A\&A, 564, A109

Sbordone, L., Monaco, L., Moni Bidin, C., et al. 2015, A\&A, 579, A104

Schlaufman, K. C., Thompson, I. B., \& Casey, A. R. 2018, ApJ, 867,98

Schlegel, D. J., Finkbeiner, D. P., \& Davis, M. 1998, ApJ, 500, 525

Schönrich, R., Binney, J., \& Dehnen, W. 2010, MNRAS, 403, 1829

Sestito, F., Longeard, N., Martin, N. F., et al. 2019, MNRAS, 484, 2166

Shapley, H. 1953, AJ, 58, 141

Sherwin, B. D., Loeb, A., \& O’Leary, R. M. 2008, MNRAS, 386, 1179

Shetrone, M., Venn, K. A., Tolstoy, E., et al. 2003, AJ, 125, 684

Skúladóttir, Á., Hansen, C. J., Salvadori, S., et al. 2019, A\&A, 631, A171

Spite, M., \& Spite, F. 1982, Nature, 297, 483

Spite, M., Cayrel, R., Plez, B., et al. 2005, A\&A, 430, 655

Spite, M., Bonifacio, P., Spite, F., et al. 2019, A\&A, 624, A44

Tissera, P. B., Scannapieco, C., Beers, T. C., et al. 2013, MNRAS, 432, 3391

Venn, K. A., Irwin, M., Shetrone, M. D., et al. 2004, AJ, 128, 1177

Venn, K. A., Kielty, C. L., Sestito, F., et al. 2020, MNRAS, 492, 3241

Wang, L., Macri, L. M., Krisciunas, K., et al. 2011, AJ, 142, 155

Wang, L., Macri, L. M., Wang, L., et al. 2013, AJ, 146, 139

Wenger, M., Ochsenbein, F., Egret, D., et al. 2000, A\&AS, 143, 9

York, D. G., Adelman, J., Anderson, J. E., et al. 2000, AJ, 120, 1579 


\section{Appendix A: Gaia identifier}

The stars we observed are not known in the SIMBAD astronomical database, except for three objects.

- GHS29 Gaia DR2 5188812082642658944, also known as CSTAR 9171, RRab type according to Gaia DR 2 (Gaia Collaboration 2018), that provides a period of 0.591620 days and an epoch of $T 0=2456935.5943$. Gaia, in the phot variable_flag also provides a "VARIABLE" flag. Gaia provides 249 visits with phot_g_mean_flux of 38449.08627837765 and phot_g_mean_flux_error of 619.2625519560296, 16\% uncertainty. The Julian date of observations is 2458784.71072 . This star was observed by Wang et al. (2011, 2013), during a seasonal observation in the Antartica winters 2008 and 2010, respectively, with the Chinese Small Telescope. They observed the star in the $\mathrm{i}$ band, and derived a variability with a period of 0.591609 days, with $T 0=55.9779(+2452500)$ in Wang et al. (2011) and 0.591726 days, with $T 0=785.4091(+2452500)$ in Wang et al. (2013). They classified it as a RR Lyr.

- GHS46 Gaia DR2 4629181692264635520, also known as $V^{*} \mathrm{Z}$ Men, a variable star of RRab type, according to Gaia DR 2, that provides a period of 0.687827 and an epoch of
$T 0=2456893.6835$. Gaia, in the phot_variable_flag, provides a "VARIABLE" flag with 146 visits. The Julian date of the observation is 2458775.7789. The star is found in Gavrilchenko et al. (2014), without classification. Demers \& Irwin (1991) provide the $\mathrm{B}$ and $\mathrm{V}$ magnitude $(V=13.85$ and $B-V=-0.09)$ in their study of stars located in the southern sky and between the Magellanic Clouds. Lopez \& Girard (1990) put this star in a sample of suspected variable stars and provide an improved position. Kukarkin et al. (1971) provide for this star a period of 0.6878 days and an RR type. This star is an object by Shapley (1953) in the article on the distribution of RR Lyr, and he provides a period of 0.688 days. Boyce (1943) studied this star in his sample of variable stars; he derived a period of 0.68781 days and put the star in a cluster.

- GHS63 Gaia DR2 2321153334969276160 is also known as CTLGM 5192. For this star, which is in the area of the open cluster Blanco 1, Platais et al. (2011) provide position and proper motion, which are consistent within the uncertainties with the values provided by Gaia DR2. They give probability zero that the star belongs to the cluster.

Table A.1. Stellar Gaia identifier.

\begin{tabular}{ll}
\hline \hline Star & Gaia designation \\
\hline GHS01 & Gaia DR2 6408116258177723776 \\
GHS02 & Gaia DR2 6406908375935227136 \\
GHS03 & Gaia DR2 6568152615142338944 \\
GHS04 & Gaia DR2 6467021184886451712 \\
GHS05 & Gaia DR2 6402407078410758144 \\
GHS06 & Gaia DR2 6459038215072888576 \\
GHS07 & Gaia DR2 6578634740526049024 \\
GHS08 & Gaia DR2 6396836437107984000 \\
GHS09 & Gaia DR2 6792492500909072768 \\
GHS10 & Gaia DR2 6375153449333298048 \\
GHS11 & Gaia DR2 6480138079431700480 \\
GHS12 & Gaia DR2 6480968592964063616 \\
GHS13 & Gaia DR2 6469971896141617408 \\
GHS14 & Gaia DR2 6779859249744647168 \\
GHS15 & Gaia DR2 6450745698376857600 \\
GHS16 & Gaia DR2 6453807460302929280 \\
GHS17 & Gaia DR2 6697340214885797248 \\
GHS18 & Gaia DR2 6472285955798151168 \\
GHS19 & Gaia DR2 6443749093572296320 \\
GHS20 & Gaia DR2 6686309364478769152 \\
GHS21 & Gaia DR2 6455330593146017664 \\
GHS22 & Gaia DR2 6425686935027126016 \\
GHS23 & Gaia DR2 6678886836357605760 \\
GHS24 & Gaia DR2 6454181878372335104 \\
GHS25 & Gaia DR2 6424093364721856640 \\
GHS26 & Gaia DR2 6673777852499865472 \\
GHS27 & Gaia DR2 4659670836211433728 \\
GHS28 & Gaia DR2 2892473389378566656 \\
GHS29 & Gaia DR2 5188812082642658944 \\
GHS30 & Gaia DR2 5401875170994688896 \\
GHS31 & Gaia DR2 5388804142405964544 \\
GHS32 & Gaia DR2 3481141194650183936 \\
GHS33 & Gaia DR2 5371147153902398080 \\
GHS34 & Gaia DR2 6186384413992963072 \\
\hline
\end{tabular}


Table A.1. continued.

\begin{tabular}{|c|c|c|}
\hline Star & Gaia designation & Other identifier \\
\hline GHS35 & Gaia DR2 6186522402702308992 & \\
\hline GHS36 & Gaia DR2 6070459535828774400 & \\
\hline GHS37 & Gaia DR2 5792409434759815680 & \\
\hline GHS38 & Gaia DR2 5819863862157033728 & \\
\hline GHS39 & Gaia DR2 6494419743340318848 & \\
\hline GHS40 & Gaia DR2 6378867354796867584 & \\
\hline GHS41 & Gaia DR2 6500170326593156352 & \\
\hline GHS42 & Gaia DR2 6344288714832612224 & \\
\hline GHS43 & Gaia DR2 6390856571321213568 & \\
\hline GHS44 & Gaia DR2 6490034581730775296 & \\
\hline GHS45 & Gaia DR2 6490954013971262976 & \\
\hline GHS46 & Gaia DR2 4629181692264635520 & $V^{*} \mathrm{Z}$ Men - variable star of RR Lyr type \\
\hline GHS47 & Gaia DR2 4817932482581995776 & \\
\hline GHS48 & Gaia DR2 4849168336616387712 & \\
\hline GHS49 & Gaia DR2 4945774589328875520 & \\
\hline GHS50 & Gaia DR2 4697867771333023744 & \\
\hline GHS51 & Gaia DR2 5008808800675101056 & \\
\hline GHS52 & Gaia DR2 5008468123868998400 & \\
\hline GHS53 & Gaia DR2 4719106247173470592 & \\
\hline GHS54 & Gaia DR2 5120933594860876544 & \\
\hline GHS55 & Gaia DR2 4709272180814135936 & \\
\hline GHS56 & Gaia DR2 4687368809680370176 & \\
\hline GHS57 & Gaia DR2 4684725274488105728 & \\
\hline GHS58 & Gaia DR2 4924385446036517760 & \\
\hline GHS59 & Gaia DR2 4901276357319875072 & \\
\hline GHS60 & Gaia DR2 4901665206478819200 & \\
\hline GHS61 & Gaia DR2 4703413673624094208 & \\
\hline GHS62 & Gaia DR2 4977325865764082688 & \\
\hline GHS63 & Gaia DR2 2321153334969276160 & CTLGM 5192 - star \\
\hline GHS64 & Gaia DR2 6567028295783009664 & \\
\hline GHS65 & Gaia DR2 6578468095795178112 & \\
\hline GHS66 & Gaia DR2 6376033402233095040 & \\
\hline GHS67 & Gaia DR2 6431108077108143488 & \\
\hline GHS68 & Gaia DR2 6363531542708636032 & \\
\hline GHS69 & Gaia DR2 5189373658205822848 & \\
\hline GHS70 & Gaia DR2 5791687571014695168 & \\
\hline GHS71 & Gaia DR2 4907295702445579776 & \\
\hline GHS72 & Gaia DR2 4983217530100818176 & \\
\hline
\end{tabular}

